\title{
High Endothelial Venules Accelerate Naive T Cell Recruitment by Tumor Necrosis Factor-Mediated R-Ras Upregulation
}

Junko Sawada, ${ }^{* \dagger}$ Carole Y. Perrot, ${ }^{* \dagger}$ Linyuan Chen, ${ }^{* \dagger}$ Ashley E. Fournier-Goss, ${ }^{* \dagger}$ Jeremiah Oyer, ${ }^{\dagger \dagger}$ Alicja Copik, ${ }^{\ddagger}$ and Masanobu Komatsu*§

From the Cancer and Blood Disorders Institute and Institute for Fundamental Biomedical Research, * Johns Hopkins All Children's Hospital, St. Petersburg, Florida; Department of Orthopaedic Surgery, ${ }^{\dagger}$ Johns Hopkins University School of Medicine, St. Petersburg, Florida; the Burnett School of Biomedical Sciences, ${ }^{\ddagger}$ University of Central Florida, Orlando Florida; and the Tumor Microenvironment and Cancer Immunology Program, ${ }^{\S}$ Sanford Burnham Prebys Medical Discovery Institute, La Jolla California

Accepted for publication October 14, 2020.

Address correspondence to Masanobu Komatsu, Ph.D., Cancer and Blood Disorders Institute, Johns Hopkins All Children's Hospital, 600 5th St. S., St. Petersburg, FL 33701. E-mail: mkomats1@jhmi.edu.

\begin{abstract}
Recruitment of naive T cells to lymph nodes is essential for the development of adaptive immunity. Upon pathogen infection, lymph nodes promptly increase the influx of naive T cells from the circulation in order to screen and prime the T cells. The precise contribution of the lymph node vasculature to the regulation of this process remains unclear. Here we show a role for the Ras GTPase, R-Ras, in the functional adaptation of high endothelial venules to increase naive $T$ cell trafficking to the lymph nodes. R-Ras is transiently up-regulated in the endothelium of high endothelial venules by the inflammatory cytokine tumor necrosis factor (TNF) within 24 hours of pathogen inoculation. TNF induces R-Ras upregulation in endothelial cells via JNK and p38 mitogen-activated protein kinase but not $\mathrm{NF}-\kappa \mathrm{B}$. Studies of T cell trafficking found that the loss of function of endothelial R-Ras impairs the rapid acceleration of naive $T$ cell recruitment to the lymph nodes upon inflammation. This defect diminished the ability of naive 0T-1 T cells to develop antitumor activity against ovalbumin-expressing melanoma. Proteomic analyses suggest that endothelial R-Ras facilitates TNF-dependent transendothelial migration (diapedesis) of naive T cells by modulating molecular assembly the at T cell-endothelial cell interface. These findings give new mechanistic insights into the functional adaptation of high endothelial venules to accelerate naive T cell recruitment to the lymph nodes. (Am J Pathol 2021, 191: 396-414; https:// doi.org/10.1016/j.ajpath.2020.10.009)
\end{abstract}

Secondary lymphoid organs, such as lymph nodes, are the primary sites for the initiation of adaptive immunity against pathogens. The initial step for adaptive immunity development is the antigen presentation to naive $\mathrm{T}$ cells by antigenpresenting cells in lymph nodes. Naive T cells are constantly recruited to lymph nodes through high endothelial venules (HEVs) for immune surveillance. ${ }^{1,2}$ However, the antigen priming of $\mathrm{T}$ cells is a rare event because naive $\mathrm{T}$ cells specific to a particular antigen of the pathogen exist at an extremely low frequency ( 1 per $10^{4}$ to $10^{6}$ naive T cells). ${ }^{2,3}$ Therefore, a rapid increase in the recruitment of naive $\mathrm{T}$ cells to the lymph nodes is necessary upon pathogen invasion in order to allow antigen-presenting cells to have greater opportunities to screen and prime the naive $\mathrm{T}$ cells and promptly develop adaptive immunity against the pathogen.

HEVs are postcapillary venules specialized for naive and memory $\mathrm{T}$ cell recruitment, which function as a gateway to the lymph nodes for these $\mathrm{T}$ cells. HEVs are found in the secondary lymphoid organs, including lymph nodes, tonsils, and Peyer's patches. ${ }^{4}$ In addition, HEVs develop in other organs and tissues in conditions with chronic inflammation, such as persistent infection, ulcerative colitis, rheumatoid

Supported by the NIH National Cancer Institute grant R01CA125255 (M.K.) and the Florida Breast Cancer Foundation (M.K.).

Disclosures: None declared. 
arthritis, and Hashimoto's thyroiditis, facilitating the formation of tertiary lymphoid structures (TLS) in these lesions. ${ }^{4}$ TLS are clusters of infiltrating immune cells, such as $\mathrm{T}$ cells, B cells, and dendritic cells, accumulating around HEVs, and they are the local sites for antigen presentation and immune activation. HEVs and TLS are also found in cancer. Their abundance correlates with patient survival and therapy responses in melanoma, pancreatic cancer, breast cancer, and sarcoma. ${ }^{5-11}$ HEVs are believed to increase $\mathrm{T}$ cell infiltration and enhance antitumor $\mathrm{T}$ cell immunity ${ }^{5-8}$; hence, they are crucial for immunotherapies, such as anti-programmed cell death 1/programmed deathligand 1 immune checkpoint blockade therapies. ${ }^{12}$

The endothelium of HEVs expresses sialomucins with 6sulfo-sialyl Lewis X carbohydrate structure, collectively known as peripheral node addressin (PNAd), on the apical surface. ${ }^{4,13}$ PNAd serves as L-selectin ligands, and together with CC-chemokines CCL19 and CCL21, it allows selective recruitment of $\mathrm{L}$-selectin ${ }^{\text {high }}$ naive and memory $\mathrm{T}$ cells. ${ }^{4} \mathrm{~A}$ series of activations of adhesion molecules and chemokine signaling allows naive $\mathrm{T}$ cells to extravasate from the blood circulation predominantly through HEVs. ${ }^{14}$ The recruitment of naive $\mathrm{T}$ cells to the lymph nodes significantly increases within the first few days of inflammation induction. ${ }^{15-18}$ This increase is crucial because the total number of cognate naive $\mathrm{CD} 4^{+} \mathrm{T}$ cells available in the lymph nodes determines the time required for the onset of Th1 immune response against pathogens. ${ }^{16}$ However, significant growth of HEVs does not occur until day 5 or later after the induction of inflammation. ${ }^{19}$ Therefore, preexisting HEVs must substantially enhance naive $\mathrm{T}$ cell trafficking to the lymph nodes in the first few days. The molecular mechanism of such a functional adaption of HEVs is unknown.

R-Ras is a Ras homolog small GTPase abundant in the endothelium of mature functional blood vessels. ${ }^{20}$ It is strongly down-regulated in the dysfunctional pathological vasculature in diabetic retinopathy ${ }^{21}$ and cancer. ${ }^{22,23}$ GAbinding protein and cAMP response element-binding proteins are known to transcriptionally regulate the RRAS gene in endothelial cells. ${ }^{24,25}$ R-Ras is a unique Ras GTPase with multifaceted roles in regulating the blood vessel morphogenesis, maturation, and stability. ${ }^{20,22,23,26-28}$ The role of RRas in HEV function is currently unknown. In this study, we generated endothelial cell-specific inducible Rras knockout (KO) mice and investigated the pathogen-induced naive $\mathrm{T}$ cell trafficking to lymph nodes to understand the precise contribution of R-Ras and the mechanism by which endothelial cells of HEVs regulate this process.

\section{Materials and Methods}

\section{Mice and Tumor Model}

All animal experiments were approved by the Institutional Animal Care and Use Committee of Johns Hopkins University. $C d h 5-\mathrm{CreER}^{\mathrm{T} 2}$ mice were obtained from Taconic
Biosciences. $\operatorname{Rras}^{\mathrm{f} / \mathrm{fl}}$ mice were generated by inGenious Targeting Laboratory (Ronkonkoma, NY) in the C57BL/6 background. We crossed $C d h 5-\mathrm{CreER}^{\mathrm{T} 2}$ mice with $\mathrm{Rras}^{\mathrm{t} / \mathrm{fl}}$ mice to generate $C d h 5-\mathrm{CreER}^{\mathrm{T} 2} ; \operatorname{Rras}^{\mathrm{t} / \mathrm{fl}}$ endothelial cell-specific tamoxifen-inducible KO (Rras-iEcKO) mice as well as Cre-negative control $\operatorname{Rras}^{\mathrm{f} / \mathrm{fl}}$ mice. A PCR primer set $5^{\prime}$-AGCATCTTGTCACTGCTGTATATAAGCCC-3' and $5^{\prime}$-CTAGCCAGATTACCATTCCC- $3^{\prime}$ was used to detect the $\operatorname{Rras}^{f l o x}$ allele. A primer set 5'-CCGGTACCAGGTCAAGTGTTAAGTG-3' and ${ }^{\prime}$ 'GCCCCTCCTGAGTCTCAATGTCTC- $3^{\prime}$ was used to detect the Cre-recombination. Primers $5^{\prime}$-GCCTGCATTACCGGTCGATGCAACGA-3' and 5'-GTGGCAGATGGCGCGGCAACACCATT-3' were used for genotyping of $C d h 5$-CreER ${ }^{\mathrm{T} 2}$. To induce Rras deletion, 6to 8-week-old Rras-iEcKO mice were injected intraperitoneally with $75 \mathrm{mg} / \mathrm{kg}$ of tamoxifen (Sigma-Aldrich, St. Louis, MO) consecutively for 5 days. The control $\operatorname{Rras}^{\mathrm{t} / \mathrm{fl}}$ mice also received tamoxifen. Rras global KO mice were described previously. ${ }^{20,22}$ C57BL/6-Tg (TcraTcrb) $1100 \mathrm{Mjb} / \mathrm{J}$ (OT-1) mice were purchased from Jackson Laboratory (Bar Harbor, ME) and maintained in our facility. Ovalbumin expressing B16F10 melanoma cells (B16F10OVA) was kindly gifted from Dr. Dmitry I. Gabrilovich from the Wistar Institute, Philadelphia, PA.

\section{CFA-Induced Inflammation}

Mice received $100 \mu \mathrm{L}$ of Complete Freund's Adjuvant (CFA; F5881; Sigma-Aldrich) into either or both the left and right hind footpads. The inguinal lymph node from each side was collected at different time points and examined individually without pooling. The volume of the lymph node (was calculated by a formula $\mathrm{d}^{2} \times \mathrm{D} / 2$, where $\mathrm{d}$ and $\mathrm{D}$ are the shortest and longest diameters, respectively). Collected lymph nodes were used for Western blot analysis, image analysis, and flow cytometry. For tumor necrosis factor (TNF) blocking, mice received $0.5 \mathrm{mg}$ of anti-TNF blocking antibody (BioXcell, clone XT3.11) or control antibody (BioXCell, clone HRPN) twice intraperitoneally 1 day before and at the same time as the intrafootpad CFA injection.

\section{Western Blot of Inguinal Lymph Node Lysate}

Inguinal lymph nodes were lysed in $0.5 \%$ SDS lysis buffer (0.5\% SDS, $0.1 \mathrm{~mol} / \mathrm{L}$ sodium phosphate buffer, $\mathrm{pH}$ 7.4) using a pestle and a $23-\mathrm{G}$ needle were used to break down the DNA aggregation. Protein concentration was measured by Pierce Modified Lowry Protein Assay Kit (Thermo Fisher Scientific, Waltham, MA). R-Ras expression levels were determined by Western blot using rabbit anti-R-Ras polyclonal antibody ${ }^{22}$ and anti-glyceraldehyde-3phosphate dehydrogenase (GAPDH) antibody (Abcam, Cambridge, United Kingdom). The band intensity was quantified by Fiji (ImageJ software version 1.53c, NIH, 
Bethesda, MD; http://imagej.nih.gov/ij) and normalized with GAPDH. Data were shown as relative values to the control group.

\section{Immunostaining and Imaging Analysis}

Lymph nodes were mounted in Tissue-Tek OCT compound, and $10-\mu \mathrm{m}$-thick frozen sections were used for immunostaining. After tissue fixation with $4 \%$ paraformaldehyde (PFA) for 10 minutes, tissue sections were blocked for nonspecific staining by $3 \%$ bovine serum albumin (BSA) and $10 \%$ goat serum/phosphate-buffered saline (PBS) for 1 hour. Anti-CD31-Alexa 548 (sc-18916 AF546; Santa Cruz Biotechnology, Dallas, TX) and/or MECA-79 Alexa 488 (sc-19602, Santa Cruz Biotechnology) were applied overnight. For R-Ras immunostaining, R-Ras antibody (clone 2E12, catalog number H00006237-M01; Abnova, Taiwan, China) was conjugated with biotin using Mix-n-Stain Biotin Antibody Labeling Kit (Biotium Inc., Fremont, CA). The sections were treated with $0.1 \%$ Triton-X 100/PBS for 10 minutes and with Avidin/Biotin Blocking Kit (SP-2001; Vector Laboratories, Burlingame, CA) followed by incubation with the primary antibody overnight and then with streptavidin-Alexa Fluor 594 or 647 conjugate (Thermo Fisher Scientific). Fluorescent images were captured by Nikon Eclipse 90i or Ti2, and image analysis was performed using NIS-Elements-Ar (Nikon, Tokyo, Japan). Sections of inguinal lymph nodes from global Rras knockout mice ${ }^{22}$ were stained with R-Ras antibody together with CD31 antibody to determine nonspecific background fluorescence intensity from the endothelium. The mean intensity of RRas staining was determined in MECA $79^{+} \mathrm{CD} 31^{+}$vessels and MECA79 ${ }^{-} \mathrm{CD} 31^{+}$vessels of wild-type animals and subtracted by the mean background fluorescence intensity in these vessels (as determined in the global Rras knockout mice). The levels of R-Ras expression in the vessels are presented in arbitrary units (AU).

For the lymph node whole-mount staining, lymph nodes were fixed with $4 \%$ PFA overnight at $4^{\circ} \mathrm{C}$, washed with PBS, and embedded in $4 \%$ low melting temperature agarose (Apex 20 to 244). The fixed lymph nodes were sectioned to 200- $\mu \mathrm{m}$ thickness using Vibratome (Thermo Fisher Scientific). The sections were incubated in $0.1 \%$ Triton-X 100 and 3\% BSA in PBS for 2 hours at room temperature to permeabilize and incubated with first antibodies overnight. The tissue sections were washed with $0.1 \%$ Triton-X 100 in PBS for 4 hours. After the incubation with secondary antibodies, the sections were washed overnight. Images were captured by confocal microscopy with the maximum projection of $\mathrm{Z}$ sections.

\section{Cell Culture and siRNA Knockdown}

Human umbilical vein endothelial cells (HUVECs), human lung microvascular endothelial cells (HMVEC-L), and human dermal microvascular endothelial cells (HMVEC-D) were purchased from Lonza (Basel, Switzerland) and maintained in EGM-2 or EGM-2MV growth media. Cells were used between 3 and 6 passages. R-Ras siRNA was custom-synthesized by Silencer siRNA that targets the RRAS sequence 5'-GGAAAUACCAGGAACAAGA-3' (Themo Fisher Scientific). Control siRNA 2 (AM4613) was obtained from Themo Fisher Scientific. Cells were transfected with siRNAs using Lipofectamine RNAi MAX Transfection Reagent (catalog number 13778030; Themo Fisher Scientific) at $10 \mathrm{nmol} / \mathrm{L}$ for 6 hours.

\section{Quantitative Real-Time PCR}

Total RNA was isolated by using Direct-zol RNA Kits (11 to 330; Genesee Scientific, San Diego, CA). cDNA was generated by High-Capacity cDNA Reverse Transcription Kit (catalog number 4368814; Thermo Fisher Scientific). TaqMan assay primers were used for gene expression analysis of RRAS (Hs00196699_m1). Primers for the PPIA gene (Hs04194521_s1) were used to determine the $2-\Delta \Delta \mathrm{Ct}$ value.

\section{Promoter Activity Assay}

The human RRAS promoter-luciferase reporter construct in a lentiviral vector, pLenti6-R4R2-RRAS-1907/+1-Fluc, was generated previously. ${ }^{25}$ HUVECs transduced with the promoter-reporter construct were cultured in 96-well plates for 24 hours at 10,000 cells per well. The medium was replaced with EGM-2 containing 0 to $10 \mathrm{ng} / \mathrm{mL} \mathrm{TNF}$, and cells were cultured for an additional 24 or 48 hours. SteadyGlo Luciferase Assay System (Promega, Madison, WI) was used to measure the promoter activity. The luminescence intensity was measured by EnVision 2105 Multimode Plate Reader (PerkinElmer, Waltham, MA). The cell content in each well was determined to normalize the luminescence intensity to the cell amount. The cell content was determined using CellTiter-Fluor Cell Viability Assay Kit (Promega).

\section{Transendothelial Migration Assay}

Human blood cells from deidentified, healthy donors were purchased from a local blood bank (OneBlood, Orlando, FL). Peripheral blood mononuclear cells (PBMCs) were separated by Ficoll-Paque Plus solution (GE Healthcare, Chicago, IL). PBMCs were aliquoted and cryopreserved before the use for experiments. HUVECs were transfected with RRAS siRNA and incubated overnight. The next day, cells were seeded onto 5 - $\mu \mathrm{m}$ pore size, 24-well Corning Transwell plates (Corning Life Sciences, Corning, NY) at $0.2 \times 10^{6}$ cells per insert and cultured for 2 days. One day before the transendothelial migration assay, cells were treated with $10 \mathrm{ng} / \mathrm{mL}$ of TNF for 24 hours. The confluent monolayer of endothelial cells was rinsed with EBM-2 basal media containing $1 \%$ BSA and used for the transendothelial 
migration assay. Human recombinant CCL21 (catalog number 366-6C-025/CF; R\&D Systems, Minneapolis, MN) was diluted to $200 \mathrm{ng} / \mathrm{mL}$ with EBM-2 containing $1 \%$ BSA and added to the lower compartment of a Transwell chamber to promote transendothelial migration of naive $\mathrm{T}$ cells. One day before the assay, vials of PBMCs were thawed and cultured in RPMI containing 1\% BSA overnight. PBMCs were labeled with $5 \mu \mathrm{M}$ carboxyfluorescein succinimidyl ester (CFSE) (catalog number 423801; Biolegend, San Diego, CA) and suspended in 1\% BSA RPMI containing 1 $\mathrm{mg} / \mathrm{mL}$ of dextran-tetramethylrhodamine(TRITC). A total of $1 \times 10^{5}$ PBMCs per well were added onto the endothelial monolayer. After 4 hours of incubation at $37^{\circ} \mathrm{C}$, the culture inserts were removed from the plates, and the plates were centrifuged at $500 \times g$ for 5 minutes to spin down the migrated $\mathrm{T}$ cells onto the plate surface. The number of CFSE-labeled cells on the plate bottom was counted by capturing multiple images of each well using a fluorescence microscope (Nikon). Separately, $200 \mu \mathrm{L}$ of supernatant from each well was transferred into another plate, and the red fluorescence intensity of dextran-TRITC was measured by a plate reader (Molecular Devices, San Jose, CA) to determine the permeability of the endothelial monolayer.

To determine the number of migrated naive $\mathrm{T}$ cells, we stained migrated cells and counted by flow cytometry. For this experiment, $4.5 \times 10^{4}$ HUVECs per well were cultured on the insert (96-well, 3- $\mu \mathrm{m}$ pore size Corning HTS Transwell) for 2 days to produce a monolayer. Nonlabeled PBMCs were added into the upper chamber at $9 \times 10^{4}$ cells per well. The cells migrated to the lower chamber were collected and stained with DURAClone T cell subset panels (catalog number B53328; Beckman Coulter, Pasadena, CA). CytoFLEX S (Beckman Coulter) was used for flow cytometry, and data were analyzed by FlowLogic version 7.3 (Miltenyi Biotec, Bergisch Gladbach, Germany). In another transendothelial migration assay, naive $\mathrm{T}$ cells were preisolated from PBMCs with an EasySep Human Naive Pan T Cell Isolation Kit (Stemcell Technologies, Vancouver, Canada). Isolated cells were labeled with $5 \mu \mathrm{mol} / \mathrm{L}$ CFSE, washed, and added into the upper chamber at $5 \times 10^{3}$ cells per well. The migrated CFSE-positive cells were collected from the lower chamber and counted by flow cytometry. The purity of isolated naive $\mathrm{T}$ cells was confirmed by flow cytometry using DURAClone $\mathrm{T}$ cell subset panels.

\section{Isolation of Naive T Cells and Adoptive Transfer to Recipient Mice}

Single-cell suspension of C57BL/6 donor mouse spleen cells was treated with BD Pharm Lyse buffer (catalog number 555899; BD Biosciences, San Jose, CA) for red cell lysis, and naive T cells were isolated using EasyStep Mouse Pan-Naïve T cell Isolation Kit (catalog number 19848; Stemcell Technologies). In this kit, nonnaive $\mathrm{T}$ cells were removed using antibodies to CD11b, CD19, CD24, CD25,
CD44, CD45R/B220, CD49b, and TER119. The purity of isolated naive $\mathrm{T}$ cells was confirmed by flow cytometry after staining cells with violetFluor 450 anti-mouse CD3 (17A2) (catalog number 75-0032-U025; Tonbo Biosciences, San Diego, CA), anti-CD44 Brilliant Violet 650 (IM7) (catalog number 103049; Biolegend), SYTOX Green Nucleic Acid Stain (catalog number S7020; Thermo Fisher Scientific), phosphatidylethanolamine (PE) anti-mouse CD62L (MEL14) (catalog number 104407; Biolegend), PerCP-Cy5.5anti-CD8a (53 to 6.7) (catalog numbe 100733; Biolegend), and APC-Cy7 anti-CD4 (RM4-5) (25-0042-U025; Tonbo Biosciences). The isolated naive $\mathrm{T}$ cells were fluorescently labeled with CellTracer Far Red Cell Proliferation Kit (catalog number C34564; Life Technologies, Carlsbad, CA). The labeled cells were intravenously injected into tail vein of CFA-inflamed recipient mice at $3 \times 10^{6}$ cell per mouse. Inguinal lymph nodes were collected 3 hours later. Single-cell suspension of each recipient lymph node was examined for the abundance of the fluorescently labeled donor naive $\mathrm{T}$ cells by flow cytometry. The trafficking of donor naive $\mathrm{T}$ cells was also examined histologically.

\section{Adoptive Transfer of Naïve 0T-1 T Cells and Analysis of Anti-Tumor Activity}

Immunization of mice with OVA-CFA mixture followed by naive OT-1 T cell transfer was performed as described, with modifications. ${ }^{29}$ Briefly, OVA peptide $\mathrm{OVA}_{257-264}$ (catalog number AS-60193-5; AnaSpec, Fremond, CA) was dissolved in PBS at $2 \mathrm{mg} / \mathrm{mL}$ and mixed with an equal volume of CFA. Rras-iEcKO and control $\operatorname{Rras}^{\mathrm{fl} / \mathrm{fl}}$ mice received 100 $\mu$ L OVA-CFA mixture (100 $\mu \mathrm{g}$ OVA) via footpad injection 24 hours before receiving naive OT-1 T cells. Naive T cells were isolated from the spleen and inguinal lymph nodes of naive OT-1 mice and intravenously transferred to the mice pre-injected with OVA-CFA at $1 \times 10^{6}$ OT- 1 T cells per mouse. Six days later, inguinal lymph nodes were collected, and the single-cell suspension of each lymph node was intravenously transferred to the second recipient mice (wildtype) bearing OVA-expressing B16F10 melanoma (B16F10OVA) implants. The tumor-bearing mice received $1 \times 10^{6}$ melanoma cells to the flanks 6 days before receiving the lymph node cell transfer. The lymph node cells from the OVA-CFA-immunized mice (average $8.6 \times 10^{6}$ cells) were transferred to each tumor-bearing mouse. Tumor size was measured by caliper and the tumor volume calculated as follows: Tumor size $\left(\mathrm{mm}^{3}\right)=\left(\right.$ Width $^{2} \times$ Length $) / 2$.

\section{Immunoprecipitation of ICAM-1 Complex}

HUVECs were treated with control or R-Ras siRNA and cultured for 2 days in a 100-mm dish. Confluent HUVEC monolayer was further cultured with or without $10 \mathrm{ng} / \mathrm{mL}$ of TNF overnight. Vials of PBMCs were thawed and cultured in RPMI containing 1\% BSA overnight. A total of $2 \times 10^{6}$ of these cells were added onto the HUVEC monolayer and 

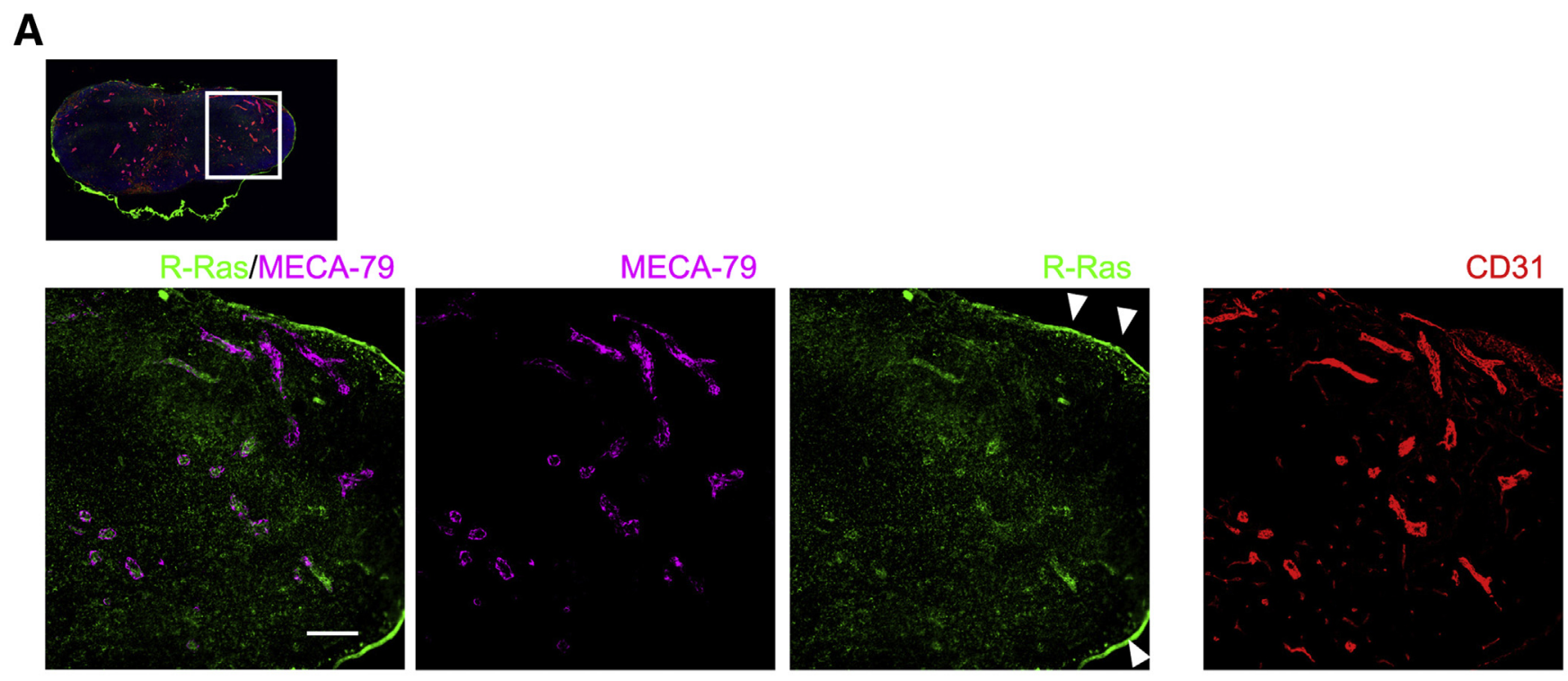

B
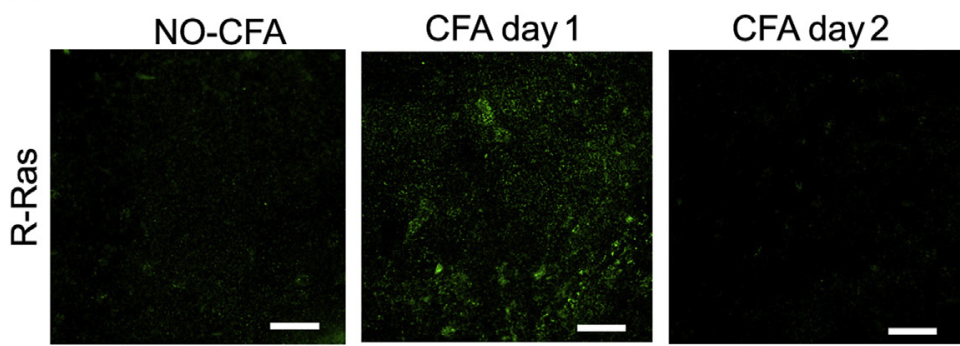

C
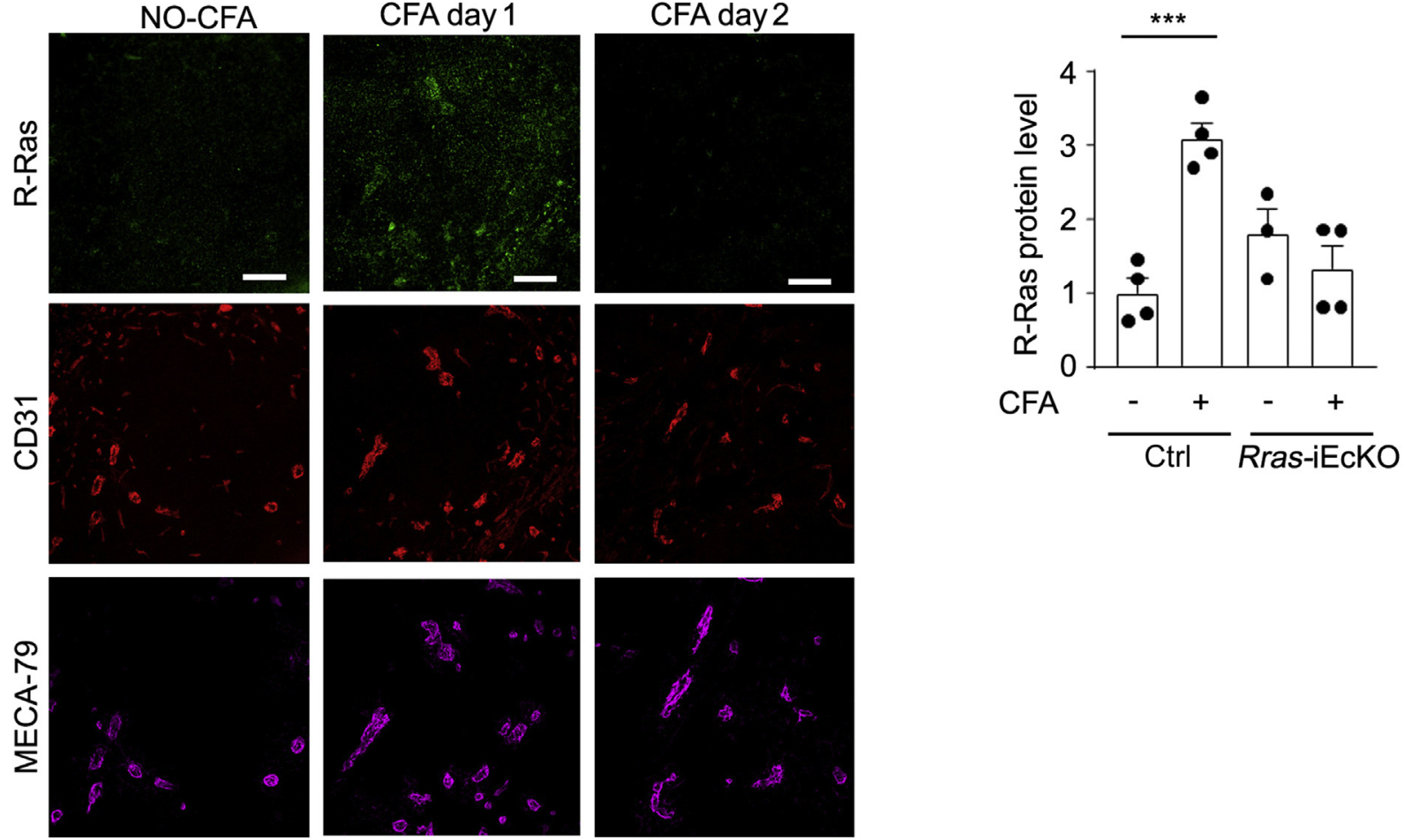

Figure 1 Inflammation upregulates R-Ras in high endothelial venules of peripheral lymph nodes. A: Immunofluorescence of R-Ras, MECA-79, and CD31 in the inguinal lymph node at 1 day after Complete Freund's Adjuvant (CFA) intrafootpad injection. Arrows indicate nonspecific tissue autofluorescence. B: Temporal pattern of R-Ras expression in the inguinal lymph node before and 1 to 2 days after CFA injection. The representative images are shown from three independent experiments. C: Western blot analysis of lymph node tissue lysate for R-Ras expression comparing endothelial cell-specific R-Ras knockout mice (Rras-iEcKO) and Cre-negative littermate control Rras ${ }^{\mathrm{fl} / \mathrm{fl}}$ mice (Ctrl). Each dot represents an inguinal lymph node from a mouse examined. Western blot was normalized by glyceraldehyde-3-phosphate dehydrogenase and shown as relative to control without CFA. Data are expressed as means \pm SEM (C). $n=3$ or 4 mice (1 lymph node was analyzed for each mouse). ${ }^{* *} P<0.001$. Scale bar $=100 \mu \mathrm{m}$.

cultured in a $\mathrm{CO}_{2}$ incubator at $37^{\circ} \mathrm{C}$ for 30 minutes. Coculture of PBMCs and HUVECs were rinsed three times with ice-cold PBS containing $\mathrm{Ca}^{2+} \mathrm{Mg}^{2+}$ to remove unbound PBMCs. The HUVEC monolayer containing the transmigrating PBMCs were lysed in NP40 cell lysis washing buffer $(1 \% \mathrm{NP} 40,50 \mathrm{mmol} / \mathrm{L} \mathrm{NaCl}, 10 \%$ glycerol, $50 \mathrm{mmol} / \mathrm{L}$ Tris $\mathrm{HCl}, \mathrm{pH}$ 7.5) with EDTA-free protease inhibitors (cOmplete; Roche, Basel, Switzerland). Cell lysate was centrifuged and supernatant was transferred into new tubes. Dynabeads M-280 sheep anti-mouse $\mathrm{IgG}$ 


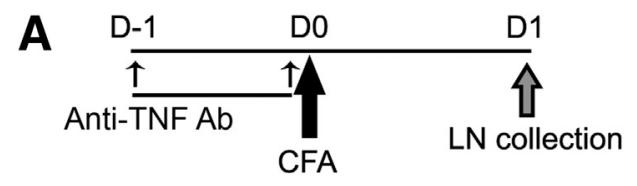

C

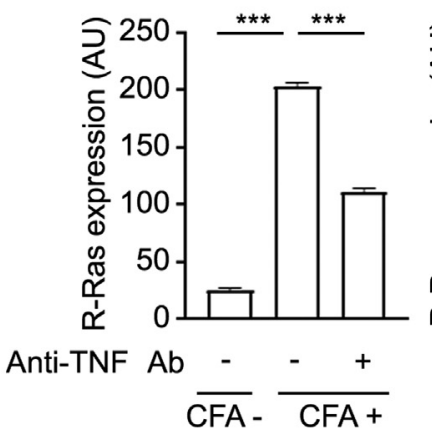

B

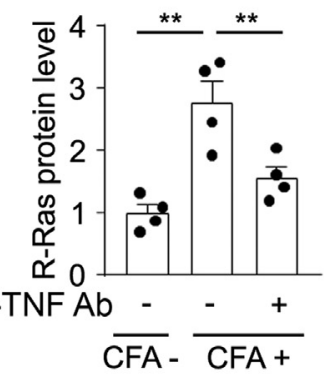

Figure 2 Tumor necrosis factor (TNF) blocking abrogates R-Ras upregulation in response to Complete Freund's Adjuvant (CFA). A: Timetable of anti-TNF antibody (Ab) administration, CFA injection, and lymph node (LN) collection. The anti-TNF Ab was administered twice to the mice. B: Western blot analysis of R-Ras protein level in the inguinal LN. One LN was examined for each mouse. Each dot represents an inguinal LN. The Western blot of each group was normalized by glyceraldehyde-3-phosphate dehydrogenase and shown as a relative value to the group without CFA and anti-TNF Ab. C: Quantitative imaging analysis of R-Ras expression in high endothelial venules (HEVs) $\left(\mathrm{MECA79}^{+}, \mathrm{CD} 31^{+}\right)$and non-HEV capillary vessels $\left(\mathrm{MECA79}{ }^{-}, \mathrm{CD} 31^{+}\right)$. LN sections $(10 \mu \mathrm{m}$ thick) were stained for R-Ras, MECA79, and CD31. The integrated fluorescence intensity of R-Ras immunostaining was determined in the two vessel types. A total of 644 to $4814 \mathrm{HEVs}$ and 3709 to 4818 non-HEV vessels were examined for R-Ras intensity. $n=4$ mice per group (B); $n=6$ LNs from 3 mice per group (C). ${ }^{* * P}<0.01,{ }^{* * *} P<0.001$.

(catalog number 11201D; Life Technologies) were incubated with anti-intercellular adhesion molecule (ICAM)-1 antibodies, clone $11 \mathrm{C} 81$ or HA58, or isotype control mouse IgG1 (MOPC-21). The supernatant of cell lysates was incubated with $60 \mu \mathrm{L}$ of antibody-bound Dynabeads for 2 hours at $4^{\circ} \mathrm{C}$. Dynabeads were washed with NP40 cell lysis buffer four times and further washed with $50 \mathrm{mmol} / \mathrm{L}$ ammonium bicarbonate buffer three times. For immunostaining of ICAM-1 complex, PBMCs were labeled with 5 $\mu \mathrm{mol} / \mathrm{L}$ CFSE before the coculture. The HUVEC monolayer culture was treated with TNF overnight and washed three times with PBS. RPMI containing $1 \%$ BSA and $100 \mathrm{ng} / \mathrm{mL}$ CCL21 was used for the coculture. After 2 hours of incubation, the HUVEC monolayer was washed twice and fixed with 4\% PFA for 10 minutes and used for ICAM-1 immunostaining.

\section{LC-MS/MS Analysis}

Immunoprecipitated proteins were digested directly on beads. Briefly, beads were thawed and resuspended with 8 $\mathrm{mol} / \mathrm{L}$ urea and $50 \mathrm{mmol} / \mathrm{L}$ ammonium bicarbonate, and cysteine disulfide bonds were reduced with $10 \mathrm{mmol} / \mathrm{L}$ tris(2-carboxyethyl)phosphine at $30^{\circ} \mathrm{C}$ for 60 minutes followed by cysteine alkylation with $30 \mathrm{mmol} / \mathrm{L}$ iodoacetamide in the dark at room temperature for 30 minutes. After alkylation, urea was diluted to $1 \mathrm{~mol} / \mathrm{L}$ urea using $50 \mathrm{mmol} /$ $\mathrm{L}$ ammonium bicarbonate, and proteins were finally subjected to overnight digestion with mass spec grade Trypsin/Lys-C mix (Promega). Finally, beads were pulled down and the solution with peptides collected into a new tube. The beads were then washed once with $50 \mathrm{mmol} / \mathrm{L}$ ammonium bicarbonate to increase peptide recovery. After digestion, samples were acidified with formic acid (FA) and subsequently desalted using AssayMap C18 cartridges mounted on an Agilent AssayMap BRAVO liquid handling system. C18 cartridges were first conditioned with $100 \%$ acetonitrile followed $0.1 \%$ FA. Samples were then loaded onto the conditioned $\mathrm{C} 18$ cartridge, washed with $0.1 \% \mathrm{FA}$, and eluted with $60 \%$ acetonitrile and $0.1 \%$ FA. Finally, the organic solvent was removed in a SpeedVac concentrator before LC-MS/MS analysis. Before the LC-MS analysis, total sample peptide amount was determined by NanoDropTM spectrophometer (Thermo Fisher Scientific).

Dried samples were reconstituted with $2 \%$ acetonitrile and $0.1 \%$ FA and analyzed by LC-MS/MS using a Proxeon EASY nanoLC system (Thermo Fisher Scientific) coupled to an Orbitrap Elite mass spectrometer (Thermo Fisher Scientific). Peptides were separated using an analytical C18 Acclaim PepMap column $(0.075 \times 500 \mathrm{~mm}, 2-\mu \mathrm{m}$ particles; Thermo Fisher Scientific) in a 120-minute gradient of $2 \%$ to $28 \%$ solvent $\mathrm{B}$ at a flow rate of $300 \mathrm{~nL} /$ minute. The mass spectrometer was operated in positive data-dependent acquisition mode. MS1 spectra were measured with a resolution of 60,000, an AGC target of $1 \mathrm{e}^{6}$, and a mass range of 350 to $1400 \mathrm{~m} / \mathrm{z}$. Up to $10 \mathrm{MS} 2$ spectra per duty cycle 
A

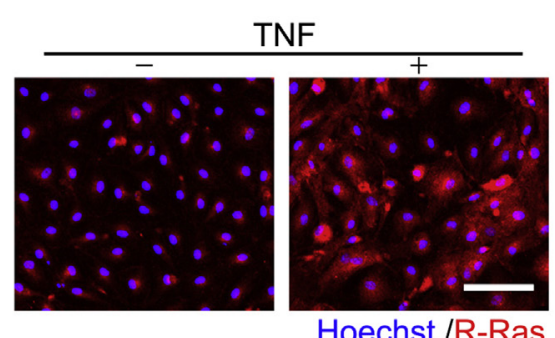

C

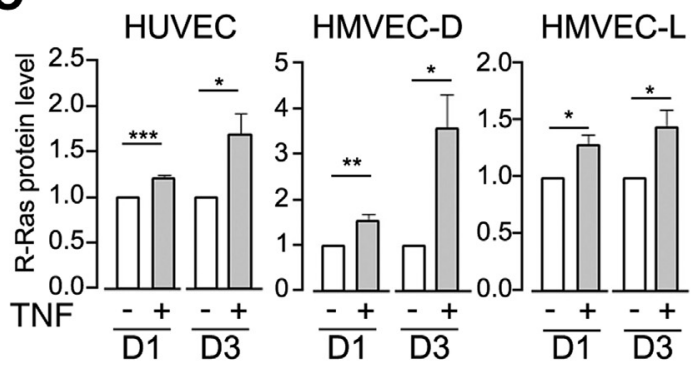

E

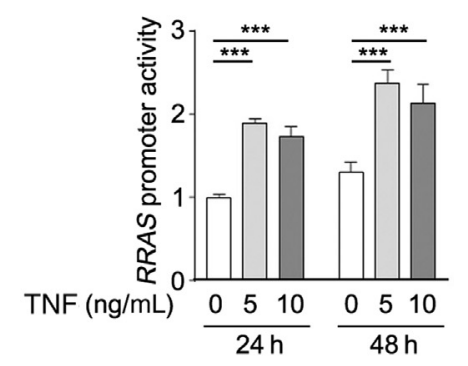

G

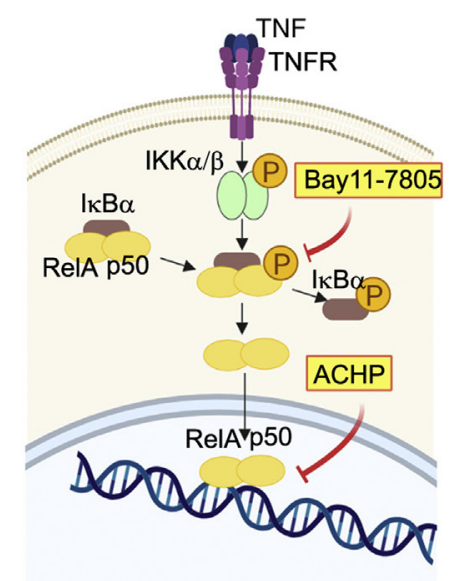

I

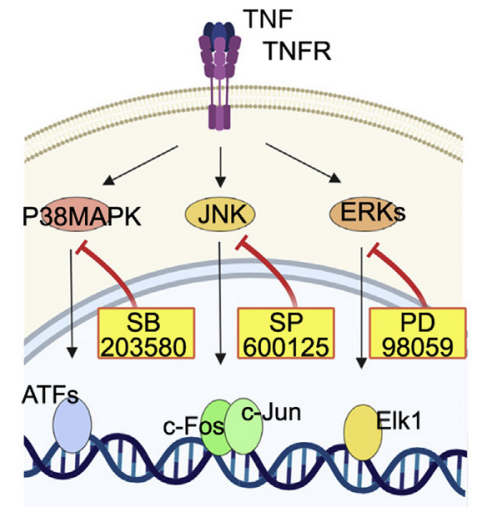

B

HUVEC HMVEC-D HMVEC-L
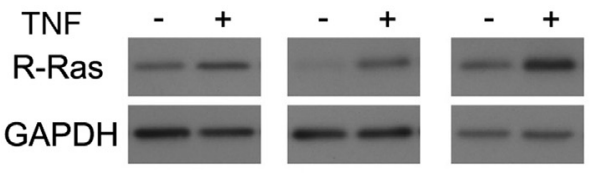

D HUVEC HMVEC-D HMVEC-L

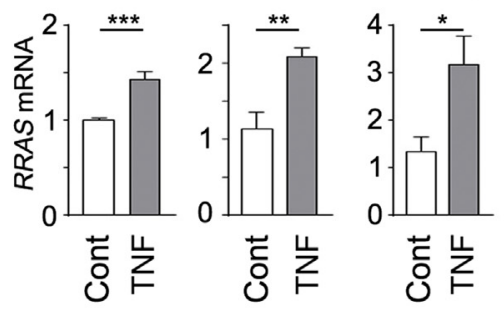

$\mathbf{F}$
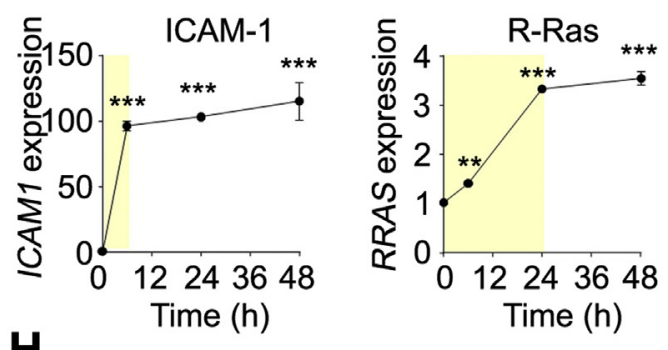

H

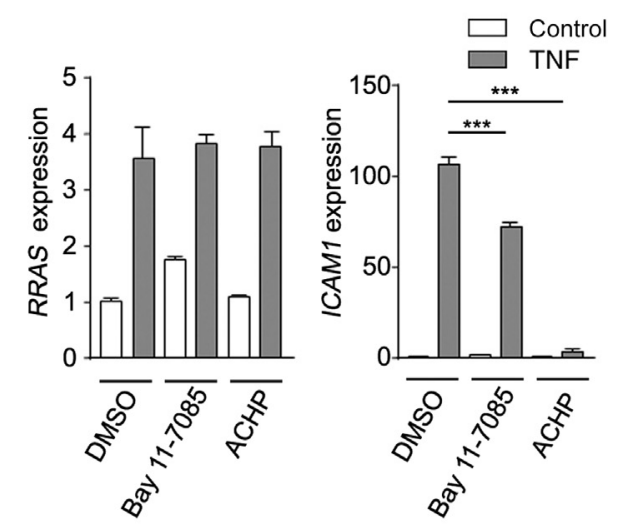

J

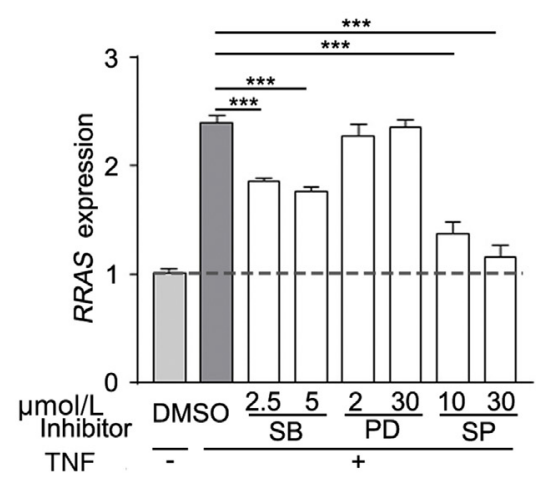

ajp.amjpathol.org - The American Journal of Pathology 
were triggered, fragmented by collisium-induced dissociation, and acquired in the ion trap with an AGC target of $1 \mathrm{e}^{4}$, an isolation window of $2.0 \mathrm{~m} / \mathrm{z}$, and a normalized collision energy of 35. Dynamic exclusion was enabled with duration of 30 seconds.

All mass spectra were analyzed with MaxQuant software version 1.5.5.1 (Max-Planck-Institute of Biochemistry, Planegg, Germany; https://www.maxquant.org). MS/MS spectra were searched against the Homo sapiens Uniprot protein sequence database (version January 2018) and GPM cRAP sequences (commonly known protein contaminants). Precursor mass tolerance was set to 20 ppm and $4.5 \mathrm{ppm}$ for the first search where initial mass recalibration was completed and for the main search, respectively. Product ions were searched with a mass tolerance $0.5 \mathrm{Da}$. The maximum precursor ion charge state used for searching was 7. Carbamidomethylation of cysteines was searched as a fixed modification, whereas oxidation of methionines and acetylation of protein $\mathrm{N}$ terminal were searched as variable modifications. Enzyme was set to trypsin in a specific mode, and a maximum of two missed cleavages was allowed for searching. The target decoy-based false discovery rate filter for spectrum and protein identification was set to $1 \%$. The MS detection intensity of each protein was normalized for the ICAM-1 intensity. For the pathway analysis, the $\log _{2}$ ratio was input to Ingenuity Pathway Analysis.

\section{Proximity Ligation Assay}

HUVECs were treated with control or R-Ras siRNA and cultured in Nunc Lab-Tek Chamber II 8 well at $5 \times 10^{4}$ cells per well for 2 days. Cells were treated with or without $10 \mathrm{ng} / \mathrm{mL}$ of TNF overnight and rinsed with 1\% BSA/RPMI three times. CFSE-labeled PBMCs were added into the chamber and incubated with $200 \mathrm{ng} / \mathrm{mL}$ CCL21 for 30 minutes. Cells were fixed with 4\% PFA for 10 minutes and used for the proximity ligation assay (PLA). Two combinations of antibodies, anti-ICAM-1 (HA58, catalog number 353101; Biolegend) and anti-Rap1b (catalog number PA5106370; Thermo Fisher Scientific) or anti-CDC42EP1 (catalog number HPA006379; Sigma-Aldrich), were used for PLA. All reagents for PLA were purchased from Sigma-
Aldrich (DUO92101-1KT), and the assay was performed following the manufacturer's instruction. Briefly, cells were permeabilized with $0.1 \%$ Triton X/PBS for 10 minutes and incubated with PLA blocking reagent for 1 hour. All antibodies were diluted at 1:100, and specimens were incubated overnight at $4 \mathrm{C}^{\circ}$. Specimens were further incubated with PLA probes for 1 hour, processed for ligation for $30 \mathrm{mi}-$ nutes, and amplified for 100 minutes at $37 \mathrm{C}^{\circ}$. Image analysis was performed with a Nikon A1R confocal microscopy and NIS-Element version AR5.21.02 software (Nikon). Three confocal images were captured for each condition. Z-stack images were taken using $40 \times$ objective lens, and 10 flames of Z-stack images at the surface of endothelial cells were used for the analysis. Endothelial cells demonstrating PLA signal surrounding the adhered PBMCs were identified and quantified as the percentage of the total number of PBMC-bound endothelial cells.

\section{Statistical Analysis}

Statistical analysis was performed by using GraphPad Prism software version 8.0.02 (GraphPad Software, San Diego, CA). A two-tailed $t$-test was used for the comparison of the two groups. The one-way analysis of variance (ANOVA) with Sidak multiple comparison test was used for multiple group comparisons. Error bars represent SEM. All experiments were repeated at least two times.

\section{Results}

Pathogen-Induced Inflammation Upregulates R-Ras in Lymph Node HEVs

We first analyzed the expression of R-Ras in the lymph nodes before and after intrafootpad inoculation of heatkilled Mycobacterium tuberculosis, known as CFA, ${ }^{30,31}$ to characterize the relationship between pathogen-induced inflammatory response and R-Ras. The CFA inoculation caused lymph node hypertrophy with a rapid fivefold increase in the lymph node weight by day 2 and an additional threefold increase from day 5 to day 10 (Supplemental Figure S1A). The first phase of expansion occurred before the growth of new HEVs, which is reported to occur at

Figure 3 Tumor necrosis factor (TNF) upregulates RRAS gene expression in cultured macrovascular and microvascular endothelial cells. A: TNF elevates R-Ras expression in human umbilical vein endothelial cells (HUVECs). Immunostaining of R-Ras in HUVECs cultured with $10 \mathrm{ng} / \mathrm{mL}$ TNF for 5 days. B-D: Western blot analysis on day 2 (B and C) and real-time quantitative RT-PCR on day 1 (D) of TNF-stimulated HUVEC, dermal microvascular human dermal microvascular endothelial cells (HMVEC-Ds), and human lung microvascular endothelial cells (HMVEC-Ls). Representative data from at least three independent experiments are shown. E: RRAS promoter activity in response to TNF was determined using HUVECs transduced with an RRAS promoter-luciferase reporter construct. Representative data are shown from two independent experiments. F: Time course analyses of RRAS and ICAM1 mRNA expression after 10-ng/mL TNF treatment. The yellow shading indicates the period before the gene expressions reach the plateau. G: A schematic diagram of the NF- $\kappa B-$ mediated TNF signaling and inhibitors Bay11-7805 and ACHP. H: Effects of the inhibitors on RRAS and ICAM1 mRNA expression in HUVECs that are stimulated or unstimulated with $10 \mathrm{ng} / \mathrm{mL}$ TNF. I: A schematic diagram of the p38 mitogen-activated protein kinase (MAPK), ERKs, and JNK-mediated TNF signaling and an inhibitor of each pathway. J: The effect of each inhibitor on RRAS mRNA expression in endothelial cells treated with or without TNF. The dotted line shows the basal level of RRAS gene expression without TNF stimulation. Data are expressed as relative values. $n=3$ (B- D, F, G, H); $n=8(\mathbf{E}) * P<0.05, * * P<0.01$, and ${ }^{* * * P}<0.001$. Scale bar $=100 \mu \mathrm{m}$. ATF, activating transcription factor; Ctrl, control; DMS0, dimethyl sulfoxide; GAPDH, glyceraldehyde-3-phosphate dehydrogenase; TNFR, tumor necrosis factor receptor. 
A

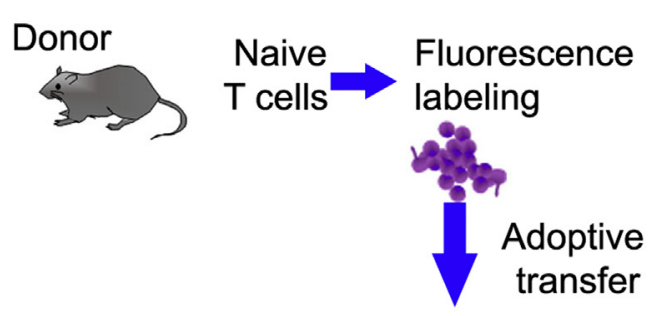

Rras-iEcKO or

Ctrl Rras ${ }^{\mathrm{fl} / \mathrm{fl}}$

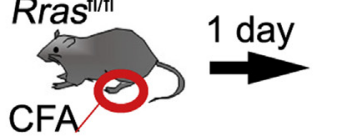

C

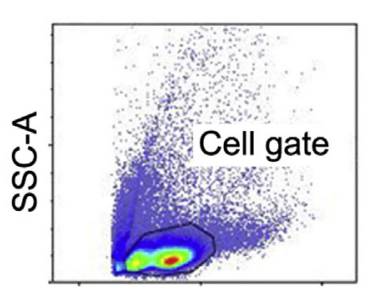

FSC-A
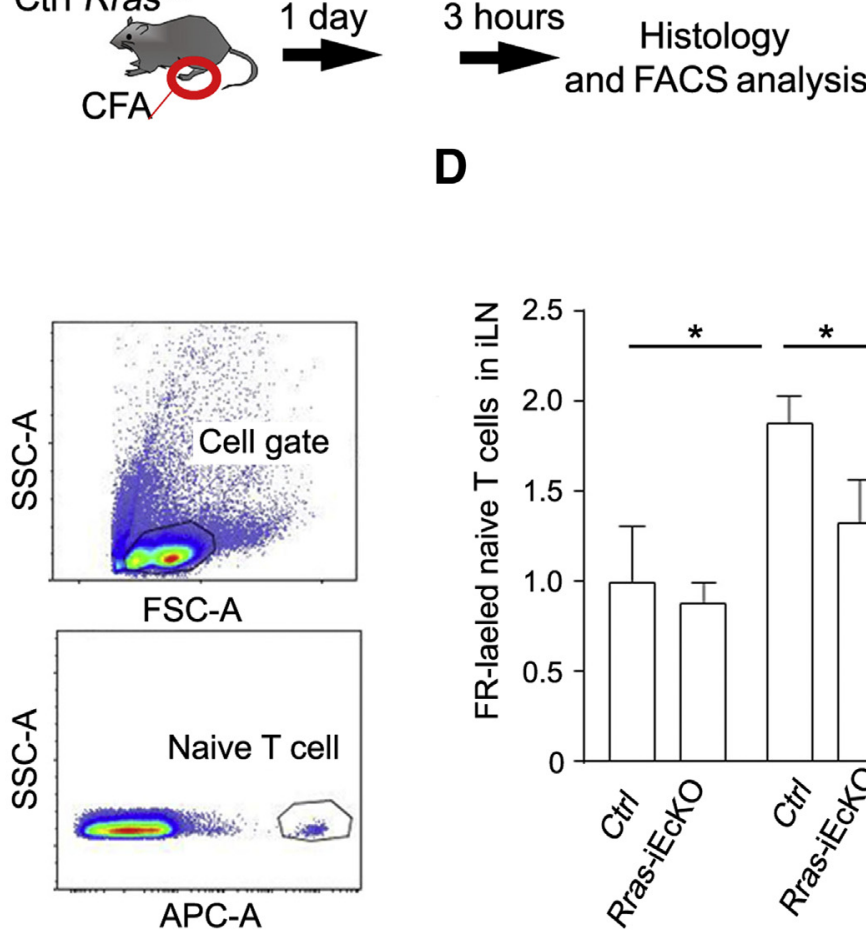

D

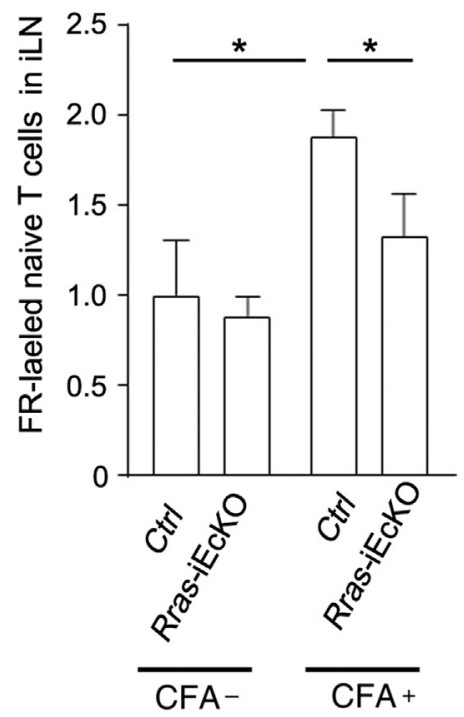

B

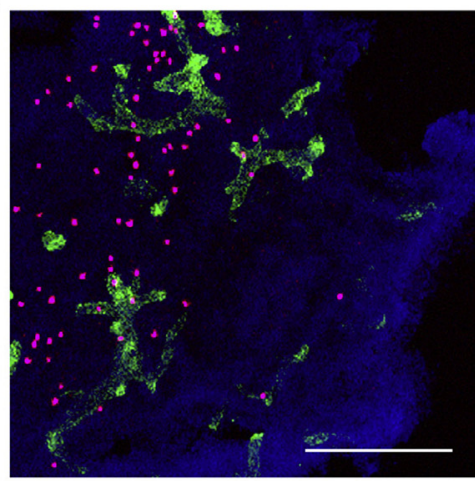

Naive T cells / MECA-79/Hoechst

E
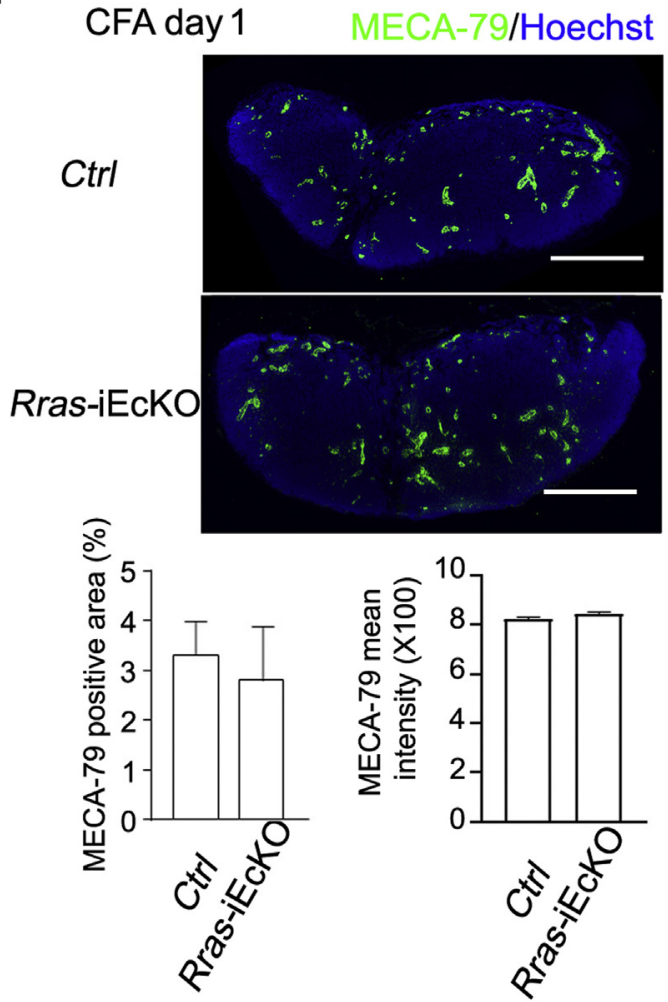

$\mathbf{F}$
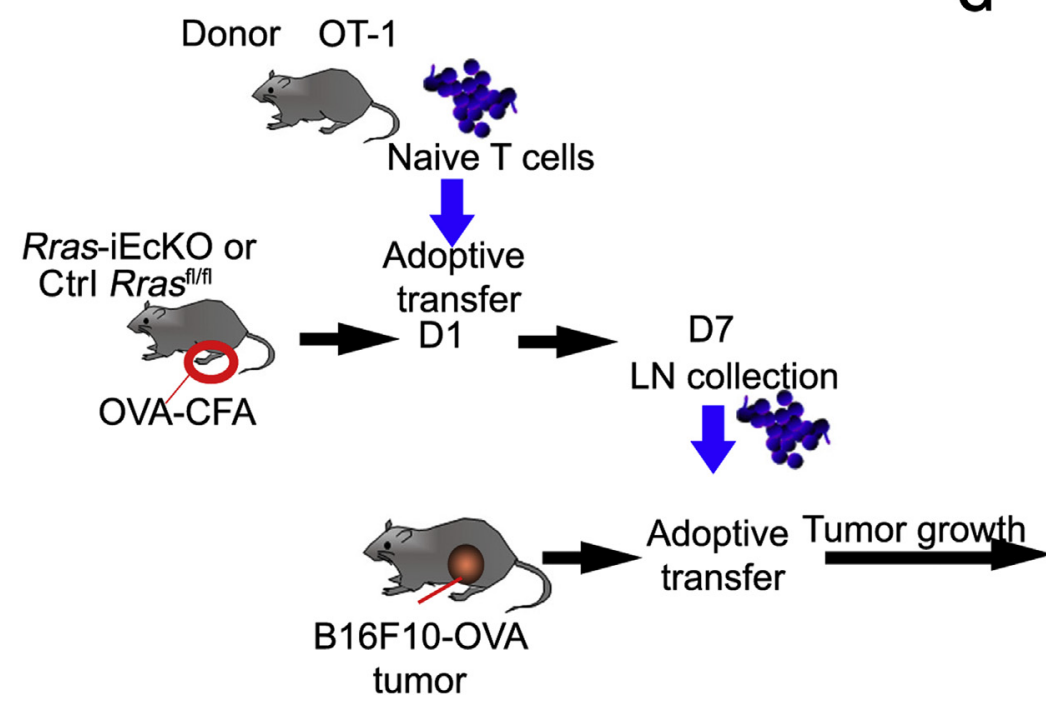

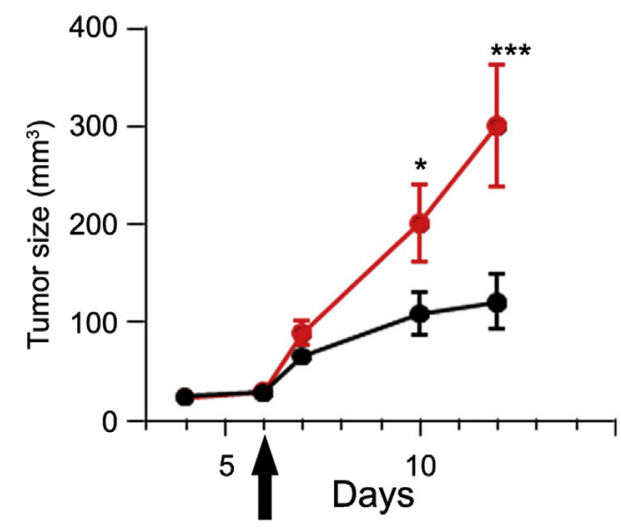

Adoptive transfer

$\rightarrow$ From control

- From Rras-iEcKO 
approximately 5 days after inflammation induction. ${ }^{19}$ A previous study found that the lymph node hypertrophy after CFA inoculation or viral infection is mainly a result of accumulation of nondividing, resting naive $\mathrm{T}$ and $\mathrm{B}$ lymphocytes. ${ }^{16}$ The nondividing $\mathrm{CD} 4^{+}$and $\mathrm{CD} 8^{+}$naive $\mathrm{T}$ cells increase substantially in the lymph nodes by day 2 , but their numbers do not increase much further until day $5 .{ }^{16}$ Because these lymphocytes are not proliferating at this stage, their accumulation is primarily attributed to the increased recruitment from the circulation and retention in the lymph nodes. ${ }^{16}$

The MECA-79 antibody recognizes PNAd, identifying the endothelium of HEVs. Interestingly, immunofluorescence analyses found a strong but transient upregulation of R-Ras in HEVs and capillary vessels 1 day after the CFA induction, which diminished on day 2 (Figure 1, A-B). Western blot of lymph node lysate also indicated the transient upregulation of R-Ras (Supplemental Figure S1B). To determine whether vascular endothelial cells are the cell type responsible for the inflammation-induced R-Ras upregulation in lymph nodes, we used endothelial cell-specific, inducible R-Ras knockout (Rras-iEcKO) mice (Supplemental Figure S2). The Rras-iEcKO and $\operatorname{Rras}^{\mathrm{f} / \mathrm{fl}}$ (Cre-negative control) mice received tamoxifen for 5 days to delete Rras in endothelial cells at 6 to 8 weeks of age. The endothelial cell-specific deletion of Rras at this age had no effect on the lymph node size (Supplemental Figure S3A), suggesting the function of noninflamed lymph node is not significantly affected. Local inflammation in the footpad was induced at 12 to 15 weeks old for the subsequent studies. The inflammation increased R-Ras expression in lymph nodes by threefolds in the control mice. By contrast, the R-Ras up-regulation was absent in the Rras-iEcKO lymph nodes (Figure 1C and Supplemental Figure S2D). These observations suggest that endothelial cells of lymph nodes up-regulate R-Ras in response to inflammation.
TNF Mediates Inflammation-Induced Endothelial R-Ras

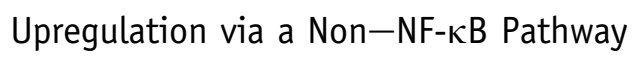

The vascular endothelium is a primary target of the proinflammatory cytokines produced by the innate immunity. In particular, TNF has a significant impact on the endothelial cell function, gene expression, and proliferation. ${ }^{32,33} \mathrm{We}$ treated mice with anti-TNF blocking antibody before and at the time of CFA injection (Figure 2A). This treatment effectively blocked the CFA-induced R-Ras upregulation in the lymph nodes (Figure 2B). Immunofluorescence of noninflamed lymph nodes had nearly undetectable R-Ras staining intensity in the capillary (MECA79 ${ }^{-} \mathrm{CD} 31^{+}$) endothelium and low but readily detectable R-Ras intensity in the HEV $\left(\mathrm{MECA}_{7}{ }^{+} \mathrm{CD} 31^{+}\right.$) endothelium (Figure 2C). The CFA inflammation caused a considerable increase in the R-Ras intensity in the endothelium of HEVs and capillary vessels. Anti-TNF blocking antibody inhibited R-Ras expression by $52 \%$ in $\mathrm{HEVs}$ and $56 \%$ in the $\mathrm{CD} 31^{+}$ endothelium, indicating that TNF is an inducer of R-Ras upregulation in the lymph node endothelium (Figure 2C).

We next tested the effect of TNF on cultured venous endothelial cells. HUVECs responded to TNF to increase RRas expression (Figures 3, A-C). Similar results were obtained for microvascular endothelial cells. TNF increased the RRAS promoter activity and mRNA levels, indicating a transcriptional regulation (Figure 3, D and E). The temporal pattern of RRAS gene induction by TNF was distinct from that of ICAMI, an essential adhesion molecule gene for $\mathrm{T}$ cell extravasation, suggesting distinct regulatory pathways for the two genes (Figure 3F). Studies with inhibitors of TNF pathway suggested that, in contrast to ICAMI, RRAS is not regulated by NF- $\kappa \mathrm{B}$, a key mediator of TNF signaling (Figure 3, G and H). TNF also activates p38 mitogenactivated protein kinase (MAPK), ERK, and JNK, although these pathways do not mediate ICAMI

\footnotetext{
Figure 4 Endothelial R-Ras deficiency impairs inflammation-induced naive T cell recruitment into lymph nodes (LNs). A: Experimental scheme for adoptive transfer of naive T cells. Recipient mice, either control (Ctrl) or Rras-iEcKO mice, received Complete Freund's Adjuvant (CFA) injection into footpads (left and right). One day after the inflammation induction when R-Ras expression is at the peak, fluorescently labeled naive T cells from wild-type donor mice were intravenously transferred to recipient mice. Left and right inguinal LNs were collected 3 hours later to determine the recruitment of naive T cells. B: Confocal microscopy shows that the transferred naive T cells (magenta) accumulated in the area surrounding MECA-79+ high endothelial venules (HEVs) (green) in the LNs. Nuclei were stained by Hoechst (blue). C: Flow cytometric detection of recruited naive T cells in the inguinal LNs. Whole LN cells were analyzed by FACS to identify fluorescently labeled naive T cells. D: Naive T cell recruitment to the inflamed LNs of Rras-iEcKO or the littermate control mice. The number of far-red fluorescence (FR)-labeled naive T cells in each inguinal LN was determined by flow cytometry and compared between different mouse groups. Data are presented relative to the Ctrl mice without CFA inflammation. Data in the graph are combined results from two independent experiments. E: Immunostaining of $\mathrm{HEV}$ s in the LNs of Rras-iEcKO or Ctrl mice. The MECA-79+ $\mathrm{HEV}$ area was determined by image analysis and presented in a graph as percentage of the total lymph node area. The mean intensity of MECA-79 staining was compared between HEVs of Rras-iEcKO and control mice. F: Experimental scheme for 0T-1 T cell transfer to tumor-bearing mice. Naive T cells were isolated from donor OT-1 transgenic mice and transferred to Rras-iEcKO or control Rras ${ }^{\mathrm{fl} / \mathrm{fl}}$ mice, which received intrafootpad inoculation of ovalbumin (OVA)-CFA mixture 1 day earlier. Six days later, inguinal LNs were isolated from these mice, and the single-cell suspension of a LN from each mouse (containing approximately $8.6 \times 10^{6}$ lymphocytes) was further transferred to a mouse harboring OVA-expressing B16F10 melanoma. G: Growth of B16F10-0VA melanoma was monitored before and after the adoptive transfer of lymphocytes from Rras-icK0 or control

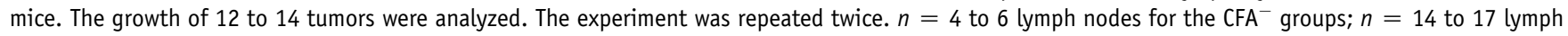
nodes for the $\mathrm{CFA}^{+}$groups (D). ${ }^{*} P<0.05,{ }^{* *} P<0.001$. Scale bars: $100 \mu \mathrm{m}$ (B); $500 \mu \mathrm{m}$ (E). AU, arbitrary unit.
} 
A

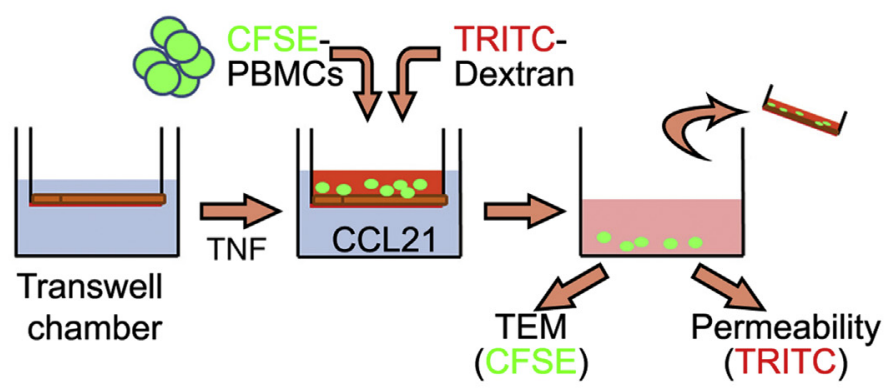

B

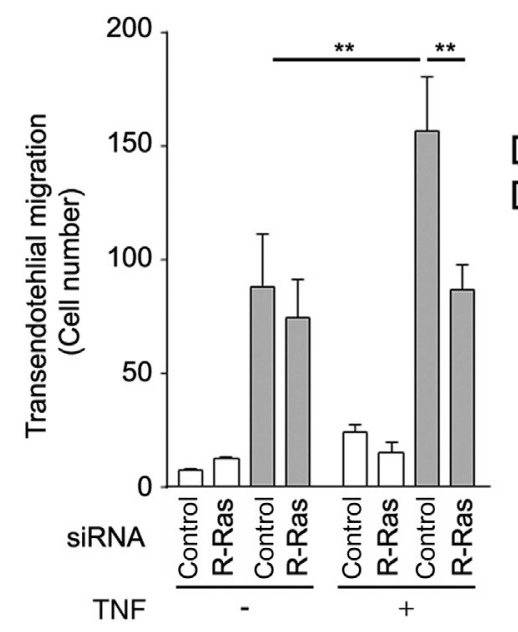

D
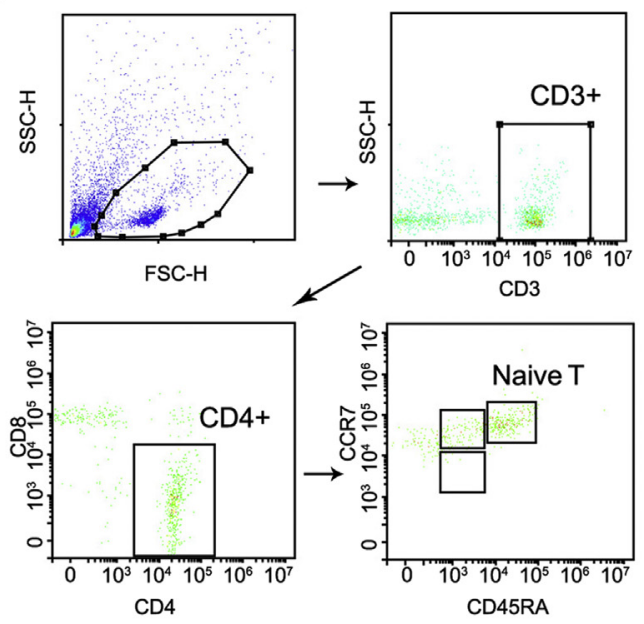

$\mathbf{F}$

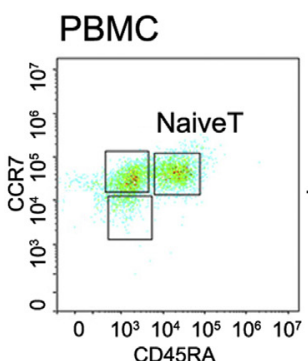

Isolated naive T cells
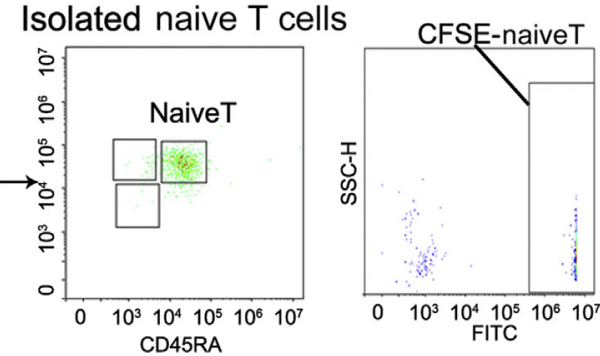

C

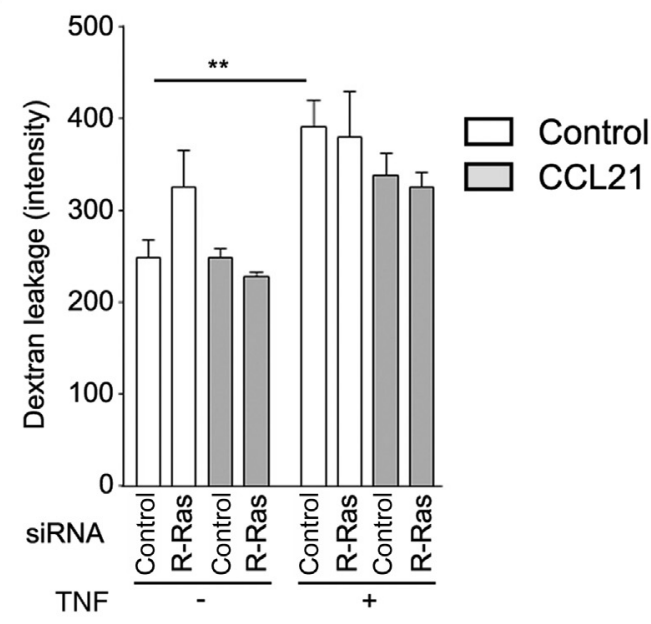

E

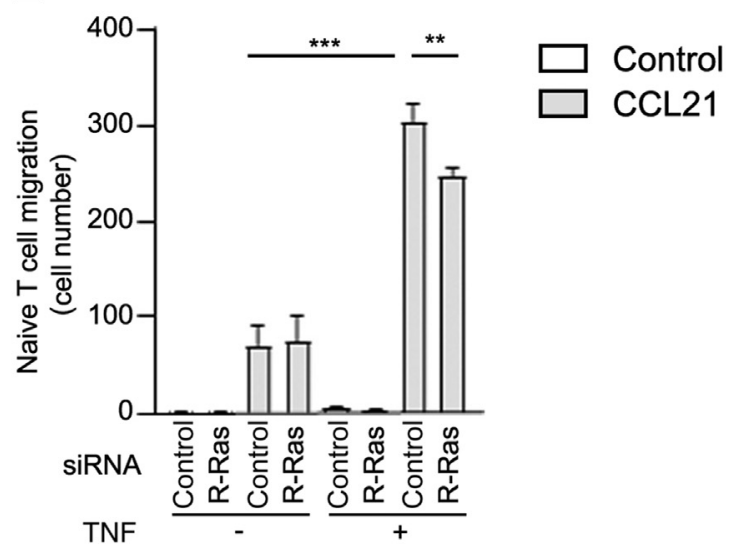


upregulation by TNF. ${ }^{34}$ In silico analyses of MotifeMap and ENCOD data suggested binding sites of Elk1, Fos/Jun, and ATF1 in the RRAS promoter region as potential target sites for these pathways (Supplemental Figure S4). Pharmacologic inhibition of JNK or p38 MAPK, but not ERK, suppressed the TNF-dependent RRAS expression, suggesting the importance of these pathways (Figure 3, I and J). These findings highlight distinct mechanisms of RRAS and ICAMI gene regulations on inflammatory stimulation of endothelial cells.

\section{Endothelial R-Ras Is Required for Acceleration of Naive T Cell Recruitment}

Upon inflammation, a prompt increase of naive $\mathrm{T}$ cell influx to lymph nodes occurs through HEVs. ${ }^{16}$ This increase occurs before the significant growth of new HEVs in the lymph nodes. ${ }^{19}$ R-Ras upregulation in HEVs coincides with this increase (Figures 1 and 2), suggesting that R-Ras may play a key role in functional adaptation of preexisting HEVs. To examine this idea, we conducted adoptive transfer of fluorescently labeled naive $\mathrm{T}$ cells from donor mice (Supplemental Figure S5), and tracked the recruitment of these $\mathrm{T}$ cells to the lymph nodes of Rras-iEcKO or control mice (Figure 4, A-D). In this study, the inflammation markedly increased the recruitment of adoptively transferred naive $\mathrm{T}$ cells into the inguinal lymph nodes in the control recipient mice as expected (Figure 4D). We confirmed that the recruited $\mathrm{T}$ cells were mainly found surrounding HEVs, consistently with the function of HEVs as a gateway for naive $T$ cells (Figure 4B). In contrast, the enhancement of naive $\mathrm{T}$ cell recruitment upon inflammation was blocked in the Rras-iEcKO recipient mice (Figure 4D). The RrasiEcKO did not affect the basal level of naive $T$ cell recruitment in the absence of inflammation (Figure 4D). MECA-79 immunofluorescence showed no significant difference in the HEV densities or PNAd expression between the lymph nodes of Rras-iEcKO and control mice (Figure 4E). The combined observations indicate that the function of HEVs to enhance the recruitment of circulating naive $T$ cells in response to inflammation is compromised by the R-Ras deficiency in endothelial cells.
Reduced Naive T Cell Recruitment due to Endothelial R-Ras Deficiency Negatively Impacts T Cell Immunity

Naive T cell recruitment to lymph nodes is an essential step for the development of $\mathrm{T}$ cell immunity. We investigated the importance of endothelial R-Ras for T cell immunity development using a syngenic tumor model expressing a specific antigen. T cells of OT- 1 transgenic mice express a $\mathrm{T}$ cell receptor that recognizes an OVA peptide $\left(\mathrm{OVA}_{257-264}\right)$ in the context of major histocompatibility complex class I $(\mathrm{H}-2 \mathrm{~Kb})$ presented by antigen-presenting dendritic cells or target cells. In this study, Rras-iEcKO and control mice were inoculated with a mixture of $\mathrm{OVA}_{257-264}$ and CFA in the footpads to induce local inflammation with the OVA antigen. The next day these mice received adoptive transfer of naive OT-1 $\mathrm{T}$ cells via intravenous injection to prime these $\mathrm{T}$ cells with the OVA antigen in the recipient's lymph nodes (Figure 4F and Supplemental Figure S5). Six days later, inguinal lymph nodes were collected from these mice, and the whole singlecell suspension of each lymph node (containing OVAprimed OT-1 T cells) was transferred to a second recipient mouse that harbors a subcutaneous B16F10-OVA tumor (Figure 4F). This experimental design allows us to determine to what degree the deficiency in R-Ras-dependent $\mathrm{T}$ cell recruitment weakens the $\mathrm{T}$ cell immunity development against antigen-presenting tumor cells. In this study, we observed a significant difference in the tumor growth rate, depending on R-Ras expression in the endothelium of the donor mice. Slow tumor growth was observed when mice received lymph node cell suspension from the OVAimmunized control mice, indicating a strong antitumor activity of the donor OT-1 T cells (Figure 4G). In contrast, rapid unattenuated tumor growth was observed when mice received lymph node cell suspension from the OVA-immunized RrasiEcKO mice (Figure 4G). This observation suggests that priming of OT-1 T cells against OVA antigen is significantly diminished in the Rras-iEcKO lymph nodes.

Because most antigen-primed effector $\mathrm{T}$ cells leave lymph nodes for the circulation within a few days, our finding suggests an involvement of OT-1 cells that differentiated to memory $\mathrm{T}$ cells in the recipient lymph nodes. To examine whether this is indeed the case, we conducted an adoptive

\footnotetext{
Figure 5 R-Ras depletion abrogates tumor necrosis factor (TNF)-mediated transendothelial migration of naïve/memory T cells to CCL21. A: A schematic diagram of transendothelial migration assay and permeability assays. A confluent monolayer of control or R-Ras-silenced human umbilical vein endothelial cells was grown on the Transwell culture insert and treated with $10 \mathrm{ng} / \mathrm{mL}$ TNF overnight. Peripheral blood mononuclear cells (PBMCs) were labeled with green fluorescence by carboxyfluorescein succinimidyl ester (CFSE) and added into the upper compartment together with tetramethylrhodamine (TRITC)-labeled 70 kDa dextran. CCL21 was added to the lower compartment to induce the migration of naive/memory T cells from the upper to lower compartment. Four hours later, the cells migrated through the endothelial monolayer were collected from the lower compartment of the chamber and counted using Nikon Element imaging software. In addition, the culture medium in the lower chamber was collected and the red fluorescence intensity was measured to determine endothelial permeability. B: Transendothelial migration of green-fluorescent cells toward CCL21 was determined for the R-Ras-silenced or control endothelial monolayer treated with or without TNF. A representative result of two independent experiments is shown. C: Dextran leakage from the upper to lower compartment was determined in the same culture groups as in B. D: Flow cytometry analysis of migrated naive T cells. Migrated cells in the bottom chamber were stained with naive T cell markers, and the naive T cell number was determined by flow cytometry. E: Comparison of naive T cell migration in different conditions. F: Naive T cells were preisolated from PBMCs, and the postsorting purity was determined by flow cytometry. G: Transendotheilal migration assay using preisolated naive T cells. Migration of CFSE-labeled preisolated naive T cells was determined by flow cytometry. Data are expressed as means \pm SEM (B-G). $n=3$ (B and C); $n=4$ wells (E). ${ }^{* *} P<0.01,{ }^{* * *} P<0.001$. FSC-H, forward scatter height; SSC-H, side scatter height.
} 
A

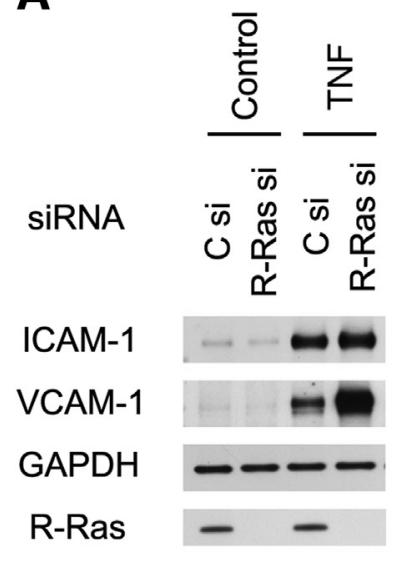

C

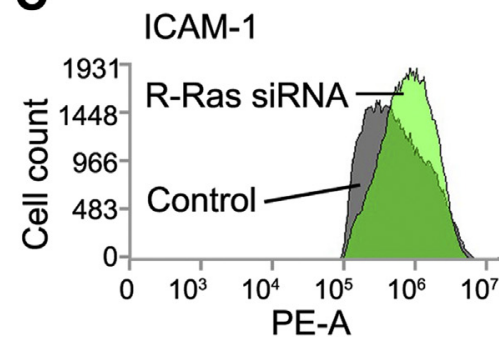

B

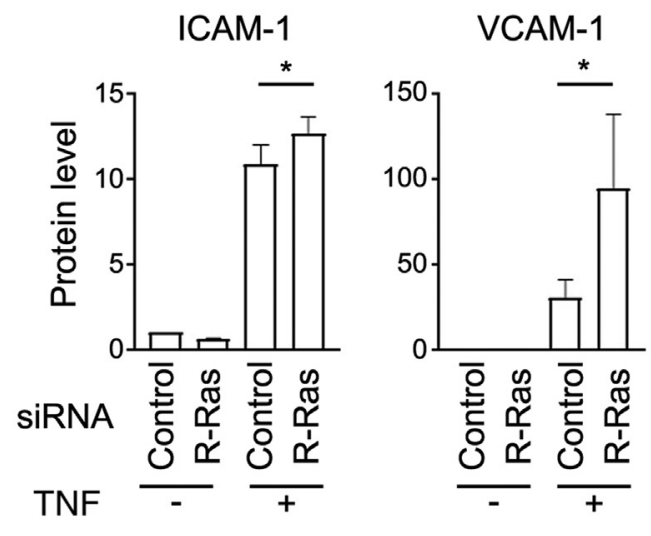

D

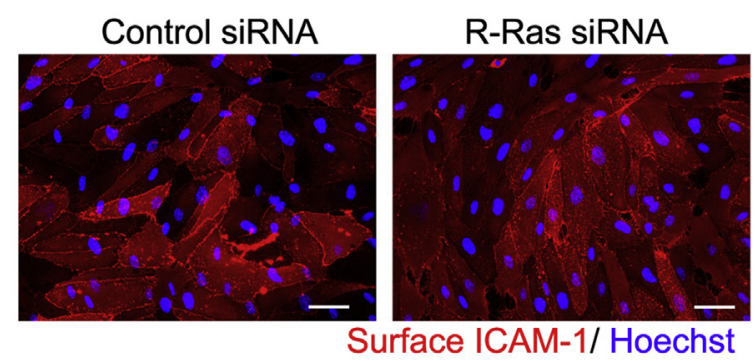

Figure 6 Expression analyses of endothelial cell adhesion molecules. A: Western blot analyses of intercellular adhesion molecule (ICAM)-1 and vascular cellular adhersion molecule (VCAM)-1 expression in the confluent culture of R-Ras-silenced or control human umbilical vein endothelial cells (HUVECs) with or without 24-hour tumor necrosis factor (TNF) stimulation. B: Western blot results were quantified and presented as relative levels of protein expression. C: FACS analysis for cell surface expression of ICAM-1. D: ICAM-1 immunofluorescence staining of nonpermeabilized HUVECs to analyze cell surface ICAM-1. $n=3$. ${ }^{*} P<0.05$. Scale bar $=50 \mu \mathrm{m}$. GAPDH, glyceraldehyde-3-phosphate dehydrogenase; PE-A, phycoerythrin area.

transfer experiment using CD45.1 congenic mice as donors of naive OT-1 T cells and tracked the differentiation of these cells to effector memory $\mathrm{T}(\mathrm{Tem})$ and central memory $\mathrm{T}(\mathrm{Tcm})$ cells in the lymph nodes of CD45.2 recipient mice at 6 days after adoptive transfer (Supplemental Figure S5C). In this analysis, we found the significant presence of donor OT-1 $\mathrm{T}$ cells that have differentiated to Tem or Tcm cells in the lymph nodes of OVA-CFA-immunized control recipient mice (Supplemental Figure S5E). In contrast, we observed a significant reduction of the number of OT-1 Tem cells in the lymph nodes of immunized Rras-iEcKO mice (Supplemental Figure S5E). There was also a trend of reduced OT-1 Tcm cells in the Rras-iEcKO lymph nodes. These results are consistent with the reduced antitumor effect of the lymph node cell transfer from the Rras-iEcKO lymph nodes (Figure 4G). However, the relative abundance of the Tem and Tcm OT-1 cells among the total OT-1 cells in the recipient lymph nodes was not affected by Rras-iEcKO, suggesting that the endothelial cell-specific R-Ras deficiency does not affect the differentiation of naive OT-1 T cells in the lymph nodes (Supplemental Figure S5F). The combined results support an important role of endothelial R-Ras in controling naive T cell recruitment to the lymph nodes, which subsequently affects the strength of adaptive immunity againsts antigens. 
R-Ras Facilitates Transendothelial Migration of Peripheral Blood Leukocytes toward CCL21

To investigate the mechanism by which endothelial R-Ras allows the accelerated recruitment of circulating naive $T$ cells, we next studied the effect of R-Ras on transmigration of these $\mathrm{T}$ cells across a confluent monolayer of endothelial cells (Figure 5A). The endothelial monolayer was stimulated with or without TNF for 24 hours to mimic the inflamed endothelium. PBMCs from healthy donors were fluorescently labeled with CFSE (green) and added to the upper chamber. The T cell migration toward CCL21 in the lower chamber was determined 4 hours later. The receptor of CCL21, CCR7, is predominantly expressed in naive T cells and memory $\mathrm{T}$ cells. ${ }^{35}$ Therefore, this assay determines the transendothelial migration of these $\mathrm{T}$ cell subtypes.

In this study, R-Ras knockdown had no effect on the transmigration of $\mathrm{T}$ cells when the endothelial monolayer was not prestimulated by TNF (Figure 5B). The TNF prestimulation markedly increased the transmigration of T cells toward CCL21. This effect of TNF was abrogated by R-Ras knockdown in the endothelial monolayer. These results demonstrated that R-Ras is necessary for the TNF-inflamed endothelial cells to increase transendothelial migration of naive/memory $\mathrm{T}$ cells in response to TNF stimulation. It is known that R-Ras enhances the endothelial barrier function by stabilizing VE-cadherin at adherens junctions and the loss of function of R-Ras increases endothelial permeability. ${ }^{22}$ Therefore, one would expect increased $\mathrm{T}$ cell migration by endothelial R-Ras knockdown. However, our result was the opposite (Figure 5B). In addition, TNF is known to increase endothelial permeability $^{36}$ as confirmed in this study by increased TRITCdextran leakage (Figure 5C). However, we found that RRas knockdown does not further increase the permeability of TNF-stimulated endothelial monolayer (Figure 5C). We also used flow cytometry to detect naive $\mathrm{T}$ cells in migrated cells and confirmed that R-Ras knockdown inhibits migration of naive $\mathrm{T}$ cells (Figure 5, D and E). In another assay, we isolated naive T cells from PBMCs, labeled with CFSE, and performed transendothelial migration assay. Migrated cells were detected by flow cytometry (Figure 5, F and G). This assay also confirmed that the naive $T$ cell migration was attenuated by R-Ras knockdown. Together, these observations indicate that $\mathrm{R}$-Ras facilitates the $\mathrm{T}$ cell migration across the TNF-inflamed endothelial wall, but this effect of $\mathrm{R}$-Ras is independent from the regulation of endothelial permeability.

\section{R-Ras Deficiency Does not Reduce Endothelial Cell Adhesion Molecules}

An inflammatory response initiated by innate immune cells, such as neutrophils, greatly elevate the expression of adhesion molecules ICAM-1 and vascular cell adhesion molecule (VCAM)-1 on the surface of endothelial cells. ${ }^{37}$
These adhesion molecules allow firm adhesion of $\mathrm{T}$ cells to endothelial cells and facilitate the subsequent extravasation of $\mathrm{T}$ cells. The R-Ras knockdown by siRNA in endothelial cells did not decrease the TNF-induced expression of the adhesion molecules (Figure 6, A-B). Instead, we observed a marked increase of VCAM-1 by R-Ras knockdown. Furthermore, the cell surface staining followed by a FACS analysis or fluorescence microscopy found no decrease of ICAM-1 on the endothelial cell surface by RRas knockdown (Figure 6, C-D). These results do not support the idea that R-Ras promotes $\mathrm{T}$ cell infiltration by enhancing ICAM-1 or VCAM-1 expression in endothelial cells upon inflammatory stimulation.

The binding of leukocyte L-selectin to PNAd expressed on the endothelial cell apical surface is responsible for the initial capturing of leukocytes from the circulation before much firmer interaction is made between the two cells. We found no apparent decrease in the PNAd expression levels in the inflamed lymph nodes of Rras-iEcKO mice as assessed by the intensity of MECA-79 immunostaining (Figure 4E). These results suggest that the impaired transendothelial migration of naive $\mathrm{T}$ cells is not caused by altered levels of endothelial adhesion molecules.

\section{R-Ras Deficiency Alters Leukocyte-Induced ICAM-1 Complex Formation in Endothelial Cells}

The transendothelial migration of leukocytes is a multistep event initiated by the leukocyte adhesion to endothelial cells. The ligation of leukocyte integrin, leukocyte function-associated antigen 1, induces ICAM-1 clustering in endothelial cells and the formation of a large molecular complex bound to the cytoplasmic tail of ICAM- $1 .{ }^{38}$ This complex is anchored to the actin cytoskeleton, creating a docking structure on the endothelial apical membrane called transmigratory cup. ${ }^{39}$ The intracellular ICAM-1 complex is composed of many molecules, including actin-binding proteins, Ras/Rho small GTPases, and kinases. The formation of this molecular complex is essential for endothelial cells to facilitate transendothelial migration of leukocytes. ${ }^{38-40}$

First, we tested whether R-Ras has an impact upon ICAM1 clustering on the adhesion of PBMCs (Supplemental Figure S6). We observed ICAM-1 clusters with strong intensity of ICAM-1 staining at the interface of PBMCs and endothelial cells, forming cup structures. However, we did not find any noticeable difference in the cup formation between R-Ras-silenced and control endothelial cells (Supplemental Figure S6). Next, we used a proteomic approach to determine how R-Ras impacts the molecular composition of the ICAM-1 complex. In this study, PBMCs were added onto the TNF-stimulated control or RRas-silenced endothelial monolayer (Figure 7A; Culture $\mathrm{b} \rightarrow \mathrm{c}$ or $\mathrm{d})$. We then immunoprecipitated ICAM-1 using two different monoclonal antibodies (Figure 7B) and identified co-precipitated proteins by LS-MS/MS protein sequencing. This study determines the proteins that are 
A

(a)

(b)

(c)

(d)

ICAM-1

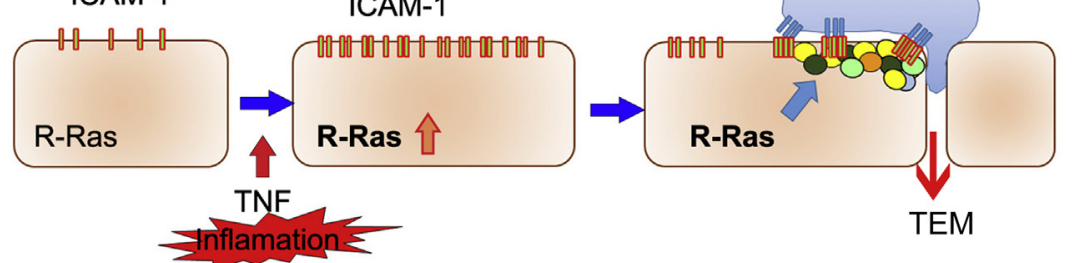

Leukocyte

ICAM-1

B

C

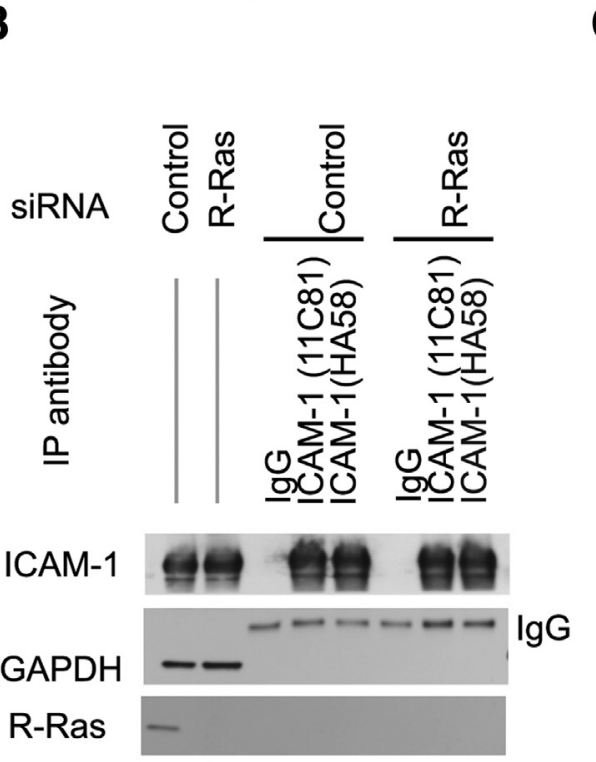

IP: ICAM-1

(b), (c), (d)

$\checkmark$

LC-MS/MS

D (c) $/($ b)

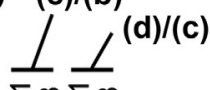

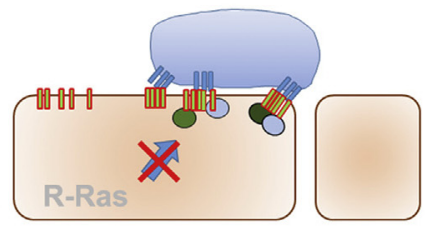

R-Ras silenced
Nomalized Intensity

Ratio

(c) $/($ b) and (d)/(c)

IPA
E

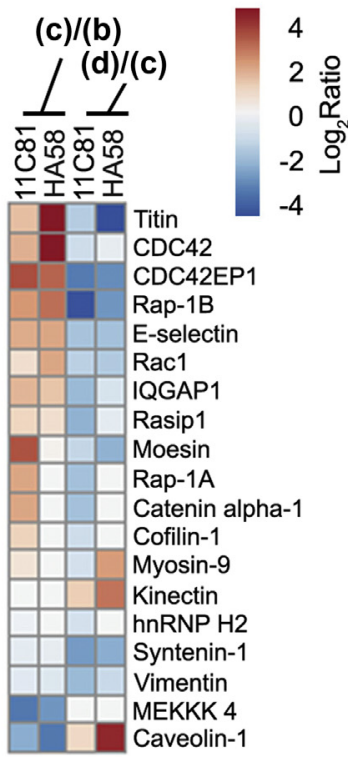

F
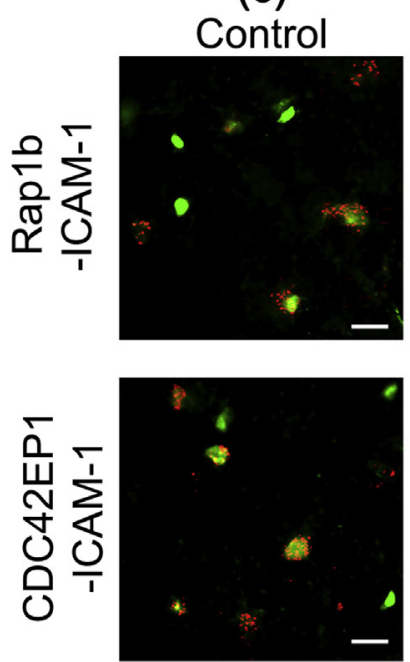

PLA signal / PBMC-CFSE
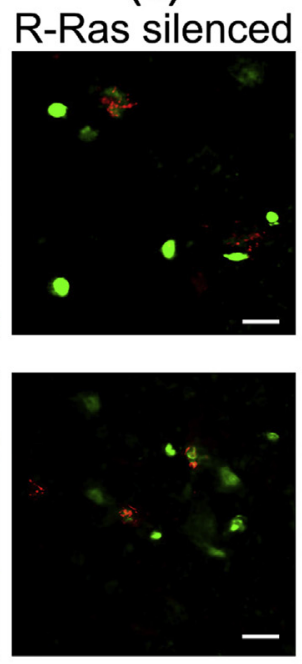

ajp.amjpathol.org
G
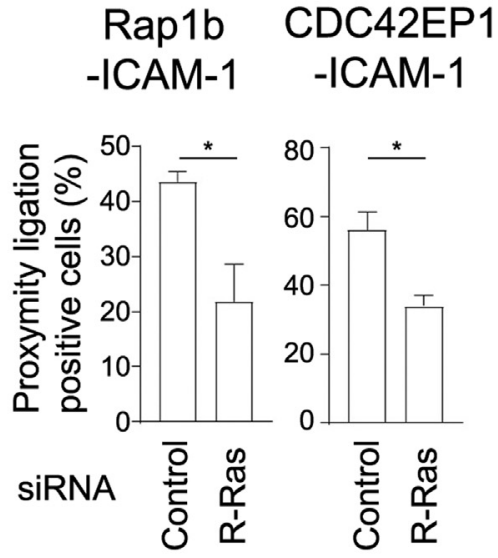
directly or indirectly associated with ICAM-1 in an RRas-dependent manner. The leukocyte function-associated antigen 1 subunits $\alpha \mathrm{L}$ and $\beta 2$ were undetected by LS-MS/ MS, suggesting that the proteins detected in the ICAM-1 immunoprecipitation are of endothelial cell origin. In addition, it is expected that the immunoprecipitated ICAM-1 mainly comes from endothelial cells because the ICAM-1 expression in PBMCs is negligible compared with that in the TNF-stimulated endothelial cells (Supplemental Figure S6C).

The normalized detection intensity of a given protein in the ICAM -1 immunoprecipitate was compared between the TNF-inflamed endothelial monolayer before and after PBMC addition (Figure 7A; Culture $\mathrm{b}$ versus $\mathrm{c}$ ), and the intensity ratio (c)/(b) was used to determine the fold change in the ICAM-1 association of the protein. A comparison was also made for the same protein between the control and $\mathrm{R}$ Ras-silenced endothelial monolayer (culture $\mathrm{c}$ versus d). The data generated here were analyzed by Ingenuity Pathway Analysis to determine the pathways that are potentially important for the leukocyte-induced ICAM-1 complex formation but negatively affected by R-Ras knockdown (Figure 7C). This analysis identified leukocyte extravasation signaling as one of the pathways that match the evaluation criteria, validating our assay strategy (Figure 7D). Other identified pathways include several pathways related to actin cytoskeleton organization and Rho family GTPase signaling, supporting the idea that R-Ras facilitates transendothelial migration of $\mathrm{T}$ cells through its effect on the actin cytoskeleton reorganization in endothelial cells.

A closer look at the individual proteins revealed that RRas knockdown in endothelial cells significantly diminishes the leukocyte-induced ICAM-1 complex association of Rap1B (Figure 7E). Other proteins that markedly diminished their association with the ICAM-1 complex are Rac1, Cdc42, Cdc42 effector protein 1 (Cdc42EP-1), Ras interacting protein 1 (Rasip), E-selectin, actin-binding ERMfamily protein moesin, and scaffold protein IQGAP1. Many of these proteins are known to be critically involved in the endothelial regulation of leukocyte transmigration process. We also performed proximity ligation assay (PLA) to confirm the association of Cdc42EP-1 and Rap1B with ICAM-1 in endothelial cells (Figure 7, F and G). In this assay, PLA signals develop when two molecules are closely associated. Conforcal imaging revealed PLA signals at the membrane periphery of the control endothelial cells in the areas surrounding the adhered PBMCs (Figure 7F), indicating the close interaction of these proteins during the cup structure formation (Supplemental Figure S6A). The number of PLA-positive endothelial cells was significantly reduced by R-Ras knockdown, indicating the importance of R-Ras for the ICAM-1 complex assembly during the cup formation (Figure 7G).

Overall, our results indicate that R-Ras is required for recruiting several important components to the ICAM-1 complex upon leukocyte adhesion to endothelial cells. Therefore, we conclude that the altered molecular composition of the ICAM-1 complex in R-Ras deficiency impairs the function of the complex as a leukocyte transportation machinery.

\section{Discussion}

One of the unsolved questions in the biology of adaptive immunity is how lymph nodes substantially increase the capacity of naive $\mathrm{T}$ cell screening and activation upon pathogen infection. In this study, we found an important role for R-Ras in increasing the naive $\mathrm{T}$ cell influx to lymph nodes by heightening endothelial function. It is well established that the robust upregulation of ICAM-1 in endothelial cells is crucial for leukocyte recruitment upon inflammation; however, the blood vessel wall cannot significantly increase the $\mathrm{T}$ cell transport in the absence of endothelial R-Ras. A previous study found that lymph nodes increase the $\mathrm{T}$ cell influx during inflammation by enlarging the diameter of primary feed arterioles for increased blood flow. ${ }^{16}$ Our

\footnotetext{
Figure 7 Proteomic analysis of intercellular adhersion molecule (ICAM)-1 complex upon leukocyte adhesion. A: A schematic diagram of the study. A confluent monolayer of human umbilical vein endothelial cells (HUVECS) expresses low levels of ICAM-1 and R-Ras (a). The endothelial monolayer was stimulated by tumor necrosis factor (TNF) for 24 hours to induce ICAM-1 and R-Ras upregulation (b). Leukocytes [peripheral blood mononuclear cells (PBMCs)] were added to the monolayer (c). The same coculture condition was prepared using R-Ras-silenced endothelial cell monolayer (d). B: The ICAM-1 complex was immunoprecipitated from the lysate of the cocultures (b, c, and d) using two different ICAM-1 antibodies, 11C81 or HA58, or control nonimmune IgG. C: The scheme of proteomic analysis. The ICAM-1 immunoprecipitate was analyzed by LC-MS/MS, and the intensity of each detected protein in the complex was normalized to the intensity of ICAM-1. The fold change between these cultures (c/b or $\mathbf{d} / \mathbf{c}$ ratio) was determined for each protein, followed by the Ingenuity Pathway Analysis (IPA). D: Results of IPA. The pathways with a set of proteins that accumulated more in the ICAM-1 complex upon leukocyte adhesion to endothelial cells are shown in higher $z$ scores in the left two columns of the heat map (c)/(b). The pathways with a set of proteins decreased in the ICAM-1 complex because of R-Ras silencing in the endothelial cells are shown in negative $z$ scores in the right two columns of the heat map (d)/(c). Asterisk indicates signaling related to leukocyte extravasation. E: Heat map of individual proteins found in the ICAM-1 complex. F: Proximity ligation assay (PLA) to determine the molecular associations. The association of ICAM-1 with Rap1b or CDC42EP1 was examined. Coculture conditions of (c) and (d) were used for this assay. The red fluorescent signal represents the close association of two molecules by proximity ligations. PBMCs were labeled with carboxyfluorescein succinimidyl ester (CFSE) (green). G: Endothelial cells demonstrating PLA signal surrounding the adhered PBMCs were identified and quantified as percentage of the total number of PBMC-bound endothelial cells. Three random areas were examined for each culture condition by confocal microscopy. Ten z-stack flames at the endothelial cell surface were used for the analysis. Data are expressed as means \pm SEM (G). ${ }^{*} P<0.05$. Scale bar $=20 \mu \mathrm{m}$. PI3K, phosphatidylinositol 3-kinase; TEM, transendothelial migration; VEGF, vascular endothelial growth factor.
} 
finding offers a mechanism that directly potentiates the lymph node vascular endothelium to increase $\mathrm{T}$ cell influx. This mechanism significantly impacts the subsequent adaptive immune response as evidenced by the decline in the ability of OT-1 T cells to suppress the growth of OVAexpressing tumors. Notably, Rras was listed as one of the genes that were significantly upregulated in HEVs upon oxazolone-induced inflammation in a recent single-cell analysis of HEV endothelial cells. ${ }^{41}$

R-Ras is a functionally unique Ras homolog GTPase that promotes blood vessel maturation and stabilization. ${ }^{20,22,23,25-28}$ In the current study, we identified a new function of R-Ras in endothelial cells to facilitate $\mathrm{T}$ cell diapidesis. This finding was unexpected but interesting because one important function of R-Ras is to limit vessel permeability by inhibiting VE-cadherin serine phosphorylation. $^{22}$ However, endothelial R-Ras deficiency decreased extravasation of circulating naive $T$ cells and transendothelial migration in vitro in inflammatory settings. This observation is consistent with the idea that $\mathrm{T}$ cell extravasation is a process that involves active transport of $\mathrm{T}$ cells by endothelial cells rather than simply a crawling of $\mathrm{T}$ cells between leaky endothelial cell-cell junctions. ${ }^{37,39}$ Although endothelial R-Ras is clearly required for the increased naive $\mathrm{T}$ cell recruitment, R-Ras upregulation was transient and lasted for only 1 day after inflammation induction (Figure 1 and Supplemental Figure S7). This finding suggests that R-Ras is important for the first response to the pathogen infection, and the homeostatic level of R-Ras expression is sufficient in the later phase of inflammation. We found that naive $T$ cell recruitment increases concurrently with R-Ras upregulation in HEVs. Dendritic cell migration into draining lymph nodes also starts early (Supplemental Figure S7). ${ }^{42}$ It is known that the majority of the recruited $\mathrm{T}$ cells in the lymph nodes are still not primed or proliferating until day 5 after HSV-2 viral infection or CFA immunization. ${ }^{16}$ This suggests that antigen-presenting dendritic cells are still going through the screening of a large amount of naive $\mathrm{T}$ cells that have been already recruited in the first 2 days, and hence, additional $\mathrm{T}$ cell recruitment would be an oversupply during this period. Supporting this idea, the numbers of recruited naive $\mathrm{CD}^{+}$and $\mathrm{CD} 8^{+} \mathrm{T}$ cells increase substantially in the first 2 days, but they do not increase much further until day 5 when these $\mathrm{T}$ cells are activated and proliferating (Supplemental Figure S7). ${ }^{16}$

It was reported that the proliferation of HEV ECs starts after day 2 to 3, and a significant expansion of HEVs is seen after day 5 (Supplemental Figure S7). ${ }^{42}$ The formation of additional HEVs depends on the increased vascular endothelial growth factor and infiltrating dendritic cells in the lymph nodes. ${ }^{42}$ However, R-Ras limits the angiogenic response of endothelial cells to vascular endothelial growth factor. $^{27,28}$ It is conceivable that the downregulation of RRas on day 2 allows the expansion of HEV endothelial cells without the inhibitory effect of R-Ras on endothelial cell proliferation. The significance of R-Ras upregulation in the capillary vessels is unknown. The circulating naive $\mathrm{T}$ cells extravasate mainly through HEVs,${ }^{16,43}$ and the accumulation of recruited naive T cells mostly around HEVs was also observed. However, the possibility that capillary-expressed $\mathrm{R}$-Ras also contributes to $\mathrm{T}$ cell recruitment in some way cannot be ruled out. R-Ras appears to be dispensable for the homeostatic leukocyte trafficking into noninflamed lymph nodes because no changes were observed in the lymph node size or transendothelial migration of leukocytes in vitro after R-Ras depletion.

As per the current understanding of leukocyte transendothelial migration, endothelial cells actively participate in this process. ICAM-1 and other adhesion molecules strap leukocytes to the endothelial cytoskeleton and facilitate the transport. This process requires ICAM-1 clustering and multiple actin-binding/organizing proteins and small GTPases to be recruited to the cluster and assemble a functional molecular complex. ${ }^{37}$ Our study of this complex showed that R-Ras is required for recruiting several of these proteins to the ICAM-1 complex. These proteins are known components of the ICAM-1 complex and/or vital to the transendothelial migration of leukocytes. For instance, Rac1 is necessary for ICAM-1 clustering, ${ }^{44}$ and Rap1B can activate Rac1 and $\mathrm{Cdc} 42 .{ }^{45-47} \mathrm{Rac} 1$ and $\mathrm{Cdc} 42$ mediate actin organization. ${ }^{48}$ E-selectin mediates initial attachment of leukocytes on endothelial cells. ${ }^{37}$ Moesin is recruited to the docking structure, anchors ICAM-1 to the actin cytoskeleton, and stabilizes the transmigratory cup. ${ }^{49}$ IQGAP knockdown in endothelial cells strongly reduces transmigration of $\mathrm{T}$ cells. ${ }^{50} \mathrm{~A}$ recent study by Arora et al ${ }^{51}$ found that IQGAP1 interacts with R-Ras and CDC42 to regulate cell membrane extensions of fibroblasts on collagen matrix, but the interaction with R-Ras was transient. IQGAP1 stabilizes the activated Rac1 and $\mathrm{Cdc} 42$ and regulates actin cytoskeleton. $^{52}$ The reduced association of Cdc42EP-1 with the ICAM-1 complex suggests that Cdc42 in the ICAM-1 complex is mostly inactive in the R-Ras deficiency. ${ }^{53}$ Interestingly, R-Ras was not detected in the ICAM-1 complex, suggesting that R-Ras regulates the assembly of ICAM-1 complex without directly interacting with it. Alternatively, R-Ras may interact with the ICAM-1 complex but only transiently so that R-Ras cannot be detected easily in the complex. The gene expression of ICAM- 1 and VCAM-1 increases in the endothelium during inflammation to increase $\mathrm{T}$ cell recruitment. R-Ras deficiency caused more substantial TNF-dependent increase of VCAM-1 expression in cultured ECs via an unknown mechanism. However, the elevated VCAM-1 did not compensate for the loss of R-Ras to facilitate leukocyte transmigration, highlighting a critical role of R-Ras in this process.

There has been an increasing interest in HEVs in recent years. Outside lymph nodes, HEVs are also found in chronic inflammatory conditions, such as rheumatoid arthritis, ulcerative colitis, and Hashimoto's thyroiditis. HEVs facilitate $\mathrm{T}$ cell infiltration to these lesions, thus augmenting the immune reaction in inflammatory diseases. On the other 
hand, the high density of intratumoral HEVs correlates with the better patient survival in various types of cancer. ${ }^{5-11}$ HEVs are thought to be critical for cancer immunotherapies, such as anti-programmed cell death protein 1/programmed death-ligand 1 immune checkpoint inhibition therapies. ${ }^{11,12}$ Our finding has a broad implication in the clinical conditions that involve the development of $\mathrm{T}$ cell immunity.

\section{Acknowledgments}

Confocal imaging was conducted at the High Resolution Imaging Core of Johns Hopkins All Children's Research Center. Histologic preparations were performed at the Histology Core, and LC-MS/MS was performed at Proteomics Core of Sanford Burnham Prebys Medical Discovery Institute.

\section{Author Contributions}

J.S. and M.K. designed the study, and J.S.,L.C. and A.E.F.G. performed experiments. C.P. performed promoter assays and analyzed results. J.O. and A.C. provided materials for experiments and performed flow cytometric assays. J.S. and M.K. analyzed results and wrote the manuscript.

\section{Supplemental Data}

Supplemental material for this article can be found at http://doi.org/10.1016/j.ajpath.2020.10.009.

\section{References}

1. Masopust D, Schenkel JM: The integration of T cell migration, differentiation and function. Nat Rev Immunol 2013, 13:309-320

2. Forster R, Braun A, Worbs T: Lymph node homing of T cells and dendritic cells via afferent lymphatics. Trends Immunology 2012, 33: $271-280$

3. Gentek R, Bajenoff M: Lymph node stroma dynamics and approaches for their visualization. Trends Immunology 2017, 38:236-247

4. Ager A: High endothelial venules and other blood vessels: critical regulators of lymphoid organ development and function. Front Immunology 2017, 8:45

5. Martinet L, Garrido I, Girard JP: Tumor high endothelial venules (HEVs) predict lymphocyte infiltration and favorable prognosis in breast cancer. Oncoimmunology 2012, 1:789-790

6. Martinet L, Le Guellec S, Filleron T, Lamant L, Meyer N, Rochaix P, Garrido I, Girard JP: High endothelial venules (HEVs) in human melanoma lesions: major gateways for tumor-infiltrating lymphocytes. Oncoimmunology 2012, 1:829-839

7. Avram G, Sanchez-Sendra B, Martin JM, Terradez L, Ramos D, Monteagudo C: The density and type of MECA-79-positive high endothelial venules correlate with lymphocytic infiltration and tumour regression in primary cutaneous melanoma. Histopathology 2013, 63: $852-861$

8. Hiraoka N, Ino Y, Yamazaki-Itoh R, Kanai Y, Kosuge T, Shimada K: Intratumoral tertiary lymphoid organ is a favourable prognosticator in patients with pancreatic cancer. Br Journal Cancer 2015, 112: $1782-1790$
9. Petitprez F, de Reynies A, Keung EZ, Chen TW, Sun CM, Calderaro J, Jeng YM, Hsiao LP, Lacroix L, Bougouin A, Moreira M, Lacroix G, Natario I, Adam J, Lucchesi C, Laizet YH, Toulmonde M, Burgess MA, Bolejack V, Reinke D, Wani KM, Wang WL, Lazar AJ, Roland CL, Wargo JA, Italiano A, Sautes-Fridman C, Tawbi HA, Fridman WH: B cells are associated with survival and immunotherapy response in sarcoma. Nature 2020, 577:556-560

10. Cabrita R, Lauss M, Sanna A, Donia M, Skaarup Larsen M, Mitra S, Johansson I, Phung B, Harbst K, Vallon-Christersson J, van Schoiack A, Lovgren K, Warren S, Jirstrom K, Olsson H, Pietras K, Ingvar C, Isaksson K, Schadendorf D, Schmidt H, Bastholt L, Carneiro A, Wargo JA, Svane IM, Jonsson G: Tertiary lymphoid structures improve immunotherapy and survival in melanoma. Nature 2020, 577:561-565

11. Helmink BA, Reddy SM, Gao J, Zhang S, Basar R, Thakur R, et al: B cells and tertiary lymphoid structures promote immunotherapy response. Nature 2020, 577:549-555

12. Allen E, Jabouille A, Rivera LB, Lodewijckx I, Missiaen R, Steri V, Feyen K, Tawney J, Hanahan D, Michael IP, Bergers G: Combined antiangiogenic and anti-PD-L1 therapy stimulates tumor immunity through HEV formation. Sci Transl Med 2017, 9: eaak9679

13. Hunter MC, Teijeira A, Halin C: T cell trafficking through lymphatic vessels. Front Immunol 2016, 7:613

14. von Andrian UH, Mempel TR: Homing and cellular traffic in lymph nodes. Nat Rev Immunol 2003, 3:867-878

15. Hay JB, Hobbs BB: The flow of blood to lymph nodes and its relation to lymphocyte traffic and the immune response. J Experimental Medicine 1977, 145:31-44

16. Soderberg KA, Payne GW, Sato A, Medzhitov R, Segal SS, Iwasaki A: Innate control of adaptive immunity via remodeling of lymph node feed arteriole. Proc Natl Acad Sci U S A 2005, 102: $16315-16320$

17. Ottaway CA, Parrott DM: Regional blood flow and its relationship to lymphocyte and lymphoblast traffic during a primary immune reaction. J Experimental Medicine 1979, 150:218-230

18. Cose SC, Jones CM, Wallace ME, Heath WR, Carbone FR: Antigenspecific CD8 $+\mathrm{T}$ cell subset distribution in lymph nodes draining the site of herpes simplex virus infection. Eur J Immunol 1997, 27: $2310-2316$

19. Kumar V, Scandella E, Danuser R, Onder L, Nitschke M, Fukui Y, Halin C, Ludewig B, Stein JV: Global lymphoid tissue remodeling during a viral infection is orchestrated by a B cell-lymphotoxindependent pathway. Blood 2010, 115:4725-4733

20. Komatsu M, Ruoslahti E: R-Ras is a global regulator of vascular regeneration that suppresses intimal hyperplasia and tumor angiogenesis. Nat Med 2005, 11:1346-1350

21. Vahatupa M, Prince S, Vataja S, Mertimo T, Kataja M, Kinnunen K, Marjomaki V, Uusitalo H, Komatsu M, Jarvinen TA, UusitaloJarvinen H: Lack of R-Ras leads to increased vascular permeability in ischemic retinopathy. Invest Ophthalmol Vis Sci 2016, 57: 4898-4909

22. Sawada J, Urakami T, Li F, Urakami A, Zhu W, Fukuda M, Li DY, Ruoslahti E, Komatsu M: Small GTPase R-Ras regulates integrity and functionality of tumor blood vessels. Cancer Cell 2012, 22: 235-249

23. Sawada J, Komatsu M: Normalization of tumor vasculature by R-Ras. Cell Cycle 2012, 11:4285-4286

24. Xu L, Komatsu M: Promoter cloning and characterization of the antivascular proliferation gene, R-ras: role of Ets- and Sp-binding motifs. J Biol Chem 2009, 284:2706-2718

25. Perrot CY, Sawada J, Komatsu M: Prolonged activation of cAMP signaling leads to endothelial barrier disruption via transcriptional repression of RRAS. FASEB J 2018, 32. fj201700818RRR

26. Li F, Sawada J, Komatsu M: R-Ras-Akt axis induces endothelial lumenogenesis and regulates the patency of regenerating vasculature. Nat Commun 2017, 8:1720 
27. Sawada J, Li F, Komatsu M: R-Ras protein inhibits autophosphorylation of vascular endothelial growth factor receptor 2 in endothelial cells and suppresses receptor activation in tumor vasculature. J Biol Chem 2015, 290:8133-8145

28. Sawada J, Li F, Komatsu M: R-Ras inhibits VEGF-induced p38MAPK activation and HSP27 phosphorylation in endothelial cells. J Vascular Research 2015, 52:347-359

29. Ke Y, Li Y, Kapp JA: Ovalbumin injected with complete Freund's adjuvant stimulates cytolytic responses. Eur J Immunol 1995, 25:549-553

30. Kumar V, Chyou S, Stein JV, Lu TT: Optical projection tomography reveals dynamics of HEV growth after immunization with protein plus CFA and features shared with HEVs in acute autoinflammatory lymphadenopathy. Front Immunol 2012, 3:282

31. Mondor I, Jorquera A, Sene C, Adriouch S, Adams RH, Zhou B, Wienert S, Klauschen F, Bajenoff M: Clonal proliferation and stochastic pruning orchestrate lymph node vasculature remodeling. Immunity 2016, 45:877-888

32. Madge LA, Pober JS: TNF signaling in vascular endothelial cells. Exp Mol Pathol 2001, 70:317-325

33. Bradley JR: TNF-mediated inflammatory disease. J Pathol 2008, 214: $149-160$

34. Zhou Z, Connell MC, MacEwan DJ: TNFR1-induced NF-kappaB, but not ERK, p38MAPK or JNK activation, mediates TNF-induced ICAM-1 and VCAM-1 expression on endothelial cells. Cell Signalling 2007, 19:1238-1248

35. Ebert LM, Schaerli P, Moser B: Chemokine-mediated control of T cell traffic in lymphoid and peripheral tissues. Mol Immunol 2005, 42:799-809

36. Mehta D, Malik AB: Signaling mechanisms regulating endothelial permeability. Physiol Reviews 2006, 86:279-367

37. Schnoor M, Alcaide P, Voisin MB, van Buul JD: Crossing the vascular wall: common and unique mechanisms exploited by different leukocyte subsets during extravasation. Mediators Inflamm 2015, 2015:946509

38. Greenwood J, Amos CL, Walters CE, Couraud PO, Lyck R, Engelhardt B, Adamson P: Intracellular domain of brain endothelial intercellular adhesion molecule-1 is essential for $\mathrm{T}$ lymphocytemediated signaling and migration. J Immunol 2003, 171:2099-2108

39. Schnoor M: Endothelial actin-binding proteins and actin dynamics in leukocyte transendothelial migration. J Immunol 2015, 194:3535-3541

40. Lyck R, Reiss Y, Gerwin N, Greenwood J, Adamson P, Engelhardt B: T cell interaction with ICAM-1/ICAM-2 double-deficient brain endothelium in vitro: the cytoplasmic tail of endothelial ICAM-1 is necessary for transendothelial migration of T cells. Blood 2003, 102:3675-3683
41. Veerman K, Tardiveau C, Martins F, Coudert J, Girard JP: Single-cell analysis reveals heterogeneity of high endothelial venules and different regulation of genes controlling lymphocyte entry to lymph nodes. Cell Rep 2019, 26:3116-31131.e5

42. Webster B, Ekland EH, Agle LM, Chyou S, Ruggieri R, Lu TT: Regulation of lymph node vascular growth by dendritic cells. J Experimental Medicine 2006, 203:1903-1913

43. Ager A, May MJ: Understanding high endothelial venules: lessons for cancer immunology. Oncoimmunology 2015, 4:e1008791

44. van Rijssel J, Kroon J, Hoogenboezem M, van Alphen FP, de Jong RJ, Kostadinova E, Geerts D, Hordijk PL, van Buul JD: The Rho-guanine nucleotide exchange factor Trio controls leukocyte transendothelial migration by promoting docking structure formation. Mol Biology Cell 2012, 23:2831-2844

45. Schlegel N, Waschke J: cAMP with other signaling cues converges on Rac1 to stabilize the endothelial barrier- a signaling pathway compromised in inflammation. Cell Tissue Res 2014, 355:587-596

46. Kurogane Y, Miyata M, Kubo Y, Nagamatsu Y, Kundu RK, Uemura A, Ishida T, Quertermous T, Hirata K, Rikitake Y: FGD5 mediates proangiogenic action of vascular endothelial growth factor in human vascular endothelial cells. Arteriosclerosis, Thrombosis, Vascular Biol 2012, 32:988-996

47. Ando K, Fukuhara S, Moriya T, Obara Y, Nakahata N, Mochizuki N: Rap1 potentiates endothelial cell junctions by spatially controlling myosin II activity and actin organization. J Cell Biol 2013, 202:901-916

48. Spiering D, Hodgson L: Dynamics of the Rho-family small GTPases in actin regulation and motility. Cell Adh Migr 2011, 5:170-180

49. Barreiro O, Yanez-Mo M, Serrador JM, Montoya MC, VicenteManzanares M, Tejedor R, Furthmayr H, Sanchez-Madrid F: Dynamic interaction of VCAM-1 and ICAM-1 with moesin and ezrin in a novel endothelial docking structure for adherent leukocytes. J Cell Biol 2002, 157:1233-1245

50. Nakhaei-Nejad M, Zhang QX, Murray AG: Endothelial IQGAP1 regulates efficient lymphocyte transendothelial migration. Eur J Immunol 2010, 40:204-213

51. Arora PD, Nakajima K, Nanda A, Plaha A, Wilde A, Sacks DB, McCulloch CA: Flightless anchors IQGAP1 and R-ras to mediate cell extension formation and matrix remodeling. Mol Biology Cell 2020, 31:1595-1610

52. Briggs MW, Sacks DB: IQGAP proteins are integral components of cytoskeletal regulation. EMBO Rep 2003, 4:571-574

53. Burbelo PD, Snow DM, Bahou W, Spiegel S: MSE55, a Cdc42 effector protein, induces long cellular extensions in fibroblasts. Proc Natl Acad Sci U S A 1999, 96:9083-9088 